Original Research Paper

\title{
Management of Renewable Energies and Environmental Protection
}

\author{
${ }^{1}$ Relly Victoria Virgil Petrescu, ${ }^{2}$ Raffaella Aversa, ${ }^{2}$ Antonio Apicella, \\ ${ }^{3}$ Samuel Kozaitis, ${ }^{4}$ Taher Abu-Lebdeh and ${ }^{1}$ Florian Ion Tiberiu Petrescu \\ ${ }^{1}$ ARoTMM-IFToMM, Bucharest Polytechnic University, Bucharest, (CE), Romania \\ ${ }^{2}$ Advanced Material Lab, Department of Architecture and Industrial Design, \\ Second University of Naples, 81031 Aversa (CE), Italy \\ ${ }^{3}$ Florida Institute of Technology, USA \\ ${ }^{4}$ North Carolina A and T State University, USA
}

Article history

Received: 27-06-2017

Revised: 02-10-2017

Accepted: 20-11-2017

Corresponding Author: Florian Ion Tiberiu Petrescu ARoTMM-IFToMM, Bucharest Polytechnic University, Bucharest, (CE) Romania Email: scipub02@gmail.com
Abstract: The purpose of this project is to present an overview of renewable energy sources, major technological developments and case studies, accompanied by applicable examples of the use of sources. Renewable energy is the energy that comes from natural resources: The wind, sunlight, rain, sea waves, tides, geothermal heat, regenerated naturally, automatically. Greenhouse gas emissions pose a serious threat to climate change, with potentially disastrous effects on humanity. The use of Renewable Energy Sources (RES) together with improved Energy Efficiency (EE) can contribute to reducing energy consumption, reducing greenhouse gas emissions and, as a consequence, preventing dangerous climate change. At least one-third of global energy must come from different renewable sources by 2050: The wind, solar, geothermal, hydroelectric, tidal, wave, biomass, etc. Oil and natural gas, classical sources of energy, have fluctuating developments on the international market. A second significant aspect is given by the increasingly limited nature of oil resources. It seems that this energy source will be exhausted in about 50 years from the consumption of oil reserves in exploitation or prospecting. "Green" energy is at the fingertips of both economic operators and individuals. In fact, an economic operator can use such a system for both own consumption and energy trading on the domestic energy market. The high cost of deploying these systems is generally depreciated in about 5-10 years, depending on the installed production capacity. The "sustainability" condition is met when projects based on renewable energy have a negative $\mathrm{CO} 2$ or at least neutral $\mathrm{CO} 2$ over the life cycle. Emissions of Greenhouse Gases (GHG) are one of the environmental criteria included in a sustainability analysis, but is not enough. The concept of sustainability must also include in the assessment various other aspects, such as environmental, cultural, health, but must also integrate economic aspects. Renewable energy generation in a sustainable way is a challenge that requires compliance with national and international regulations. Energy independence can be achieved: - Large scale (for communities); - small-scale (for individual houses, vacation homes or cabins without electrical connection).

Keywords: Environmental Protection, Renewable Energy, Sustainable Energy, The Wind, Sunlight, Rain, Sea Waves, Tides, Geothermal Heat, Regenerated Naturally

(C) 2017 Relly Victoria Virgil Petrescu, Raffaella Aversa, Antonio Apicella, Samuel Kozaitis, Taher Abu-Lebdeh and Florian Ion Tiberiu Petrescu. This open access article is distributed under a Creative Commons Attribution (CC-BY) 3.0 


\section{Introduction}

The purpose of this project is to present an overview of renewable energy sources, major technological developments and case studies, accompanied by applicable examples of the use of sources.

Renewable energy is the energy that comes from natural resources: The wind, sunlight, rain, sea waves, tides, geothermal heat, regenerated naturally, automatically.

Greenhouse gas emissions pose a serious threat to climate change, with potentially disastrous effects on humanity. The use of Renewable Energy Sources (RES) together with improved Energy Efficiency (EE) can contribute to reducing energy consumption, reducing greenhouse gas emissions and, as a consequence, preventing dangerous climate change.

At least one-third of global energy must come from different renewable sources by 2050: The wind, solar, geothermal, hydroelectric, tidal, wave, biomass, etc.

Oil and natural gas, classical sources of energy, have fluctuating developments on the international market. A second significant aspect is given by the increasingly limited nature of oil resources. It seems that this energy source will be exhausted in about 50 years from the consumption of oil reserves in exploitation or prospecting.

"Green" energy is at the fingertips of both economic operators and individuals.

In fact, an economic operator can use such a system for both own consumption and energy trading on the domestic energy market. The high cost of deploying these systems is generally depreciated in about 5-10 years, depending on the installed production capacity.

The "sustainability" condition is met when projects based on renewable energy have a negative $\mathrm{CO} 2$ or at least neutral $\mathrm{CO} 2$ over the life cycle.

Emissions of Greenhouse Gases (GHG) are one of the environmental criteria included in a sustainability analysis, but is not enough. The concept of sustainability must also include in the assessment various other aspects, such as environmental, cultural, health, but must also integrate economic aspects.

Renewable energy generation in a sustainable way is a challenge that requires compliance with national and international regulations.

Energy independence can be achieved:

- Large scale (for communities)

- Small-scale (for individual houses, vacation homes or cabins without electrical connection)

Today, the renewable energy has gained an avantgarde and a great development also thanks to governments and international organizations that have finally begun to understand its imperative necessity for humanity, to avoid crises and wars, to maintain a modern life (we can't go back to caves).

\section{Materials and Methods}

\section{Solar Energy}

Solar energy means the energy that is directly produced by the transfer of light energy radiated by the Sun into other forms of energy. This can be used to generate electricity or to heat the air and water. Although solar energy is renewable and easy to produce, the main problem is that the sun does not provide constant energy over a day, depending on the day-night alternation, weather conditions, season.

Solar Panels generate electricity approx. 9h/day (the calculation is minimal, the winter is $9 \mathrm{~h}$ ), feeding the consumers and charging the batteries at the same time.

Solar installations are of two types: Thermal and photovoltaic.

Photovoltaics produce electricity directly, thermal ones help save $75 \%$ of other fuels (wood, gas) per year. A house that has both solar installations (with photovoltaic and vacuum thermal panels) can be considered "energy independence" (because the energy accumulated in the day is then sent to the grid and used as needed).

The use of solar radiation for the production of electricity can be done by several methods:

(1) The use of photovoltaic modules - by capturing the energy of the photons coming from the sun and storing it in free electrons, thereby generating an electric current, solar photovoltaic panels generating electricity

(2) The use of solar towers

(3) Using Parabolic Concentrators - This type of concentrator consists of a gutter-shaped parabolic mirror that concentrates solar radiation on a pipe. A working fluid is circulating in the duct which is generally an oil that takes up the heat to give it water to produce the steam that drives the turbine of an electric generator. The concentrator requires adjusting the posture position of the sun in the apparent daytime displacement

(4) Using the Dish-Stirling system

Solar installations work even when the sky is dark. They are also resistant to hail (in the case of the best panels; the Fig. 1).

Solar-thermal systems are mainly made with flatbottomed solar collectors or vacuum tubes, especially for smaller solar radiation in Europe. In the energy potential assessments, applications concerning water heating or enclosures/swimming pools (domestic hot water, heating, etc.) were considered.

Locations for solar-thermal applications (thermal energy).

In this case, any available space can be used if:

- Allows the location of solar thermal collectors 
- Preferential orientation to the South and inclination according to location latitude

This is the case for roofs of houses/blocks (Fig. 2), adjacent buildings (covered parking lots, etc.) or land on which solar-thermal collectors can be located (Aversa et al., 2017 a-d; 2016 a-d; Petrescu et al., 2016 a-b; Mirsayar et al, 2017; Blue Planet; World Tree, From Wikipedia; Giovanni et al., 2012).

For the solar photovoltaic potential, both photovoltaic power grid applications and autonomous (non-grid) applications for isolated consumers were considered.

Solar energy can be used very easily - with a photovoltaic system. This type of system transforms the sunlight into electricity throughout the year, with the point that only high-quality photovoltaic systems that produce electricity over a long time are profitable (Fig. 3). The system also allows other energy sources to be coupled with solar energy such as wind energy produced by a turbine. Obviously, besides the converter, it is also necessary to have a battery that is strong enough to retain as much energy as possible during the night, or when the consumption amount is very low and to release it when necessary. A system for producing, distributing and maintaining renewable energies for a house, cottage, motel, hospital, even located in isolated places, where the power grid does not reach, is presented in the figure 3. If the wind does not blow in a long period and the sky is not sunny, it is necessary to have an electric generator inserted into the system.

\section{Wind Potential}

The winds are due to the fact that the Earth's equatorial regions receive more solar radiation than the polar regions, thus creating a large number of convection currents in the atmosphere. According to meteorological assessments, about $1 \%$ of the solar input is converted to wind energy, while $1 \%$ of the daily wind energy contribution is roughly equivalent to the world's daily energy consumption. This means that global wind resources are in large, widespread quantities. More detailed assessments are needed to quantify resources in certain areas.

Wind energy production began very early centuries ago, with sailboats, windmills and grain mowers. It was only at the beginning of this century that high-speed wind turbines were developed to generate electricity. The term wind turbine is widely used today for a rotating blade machine that converts the kinetic energy of the wind into useful energy. Currently there are two categories of base wind turbines: Wind turbine Wind Turbines (HAWT) and Vertical Wind Turbines (VAWT), depending on the axis orientation of the rotor.

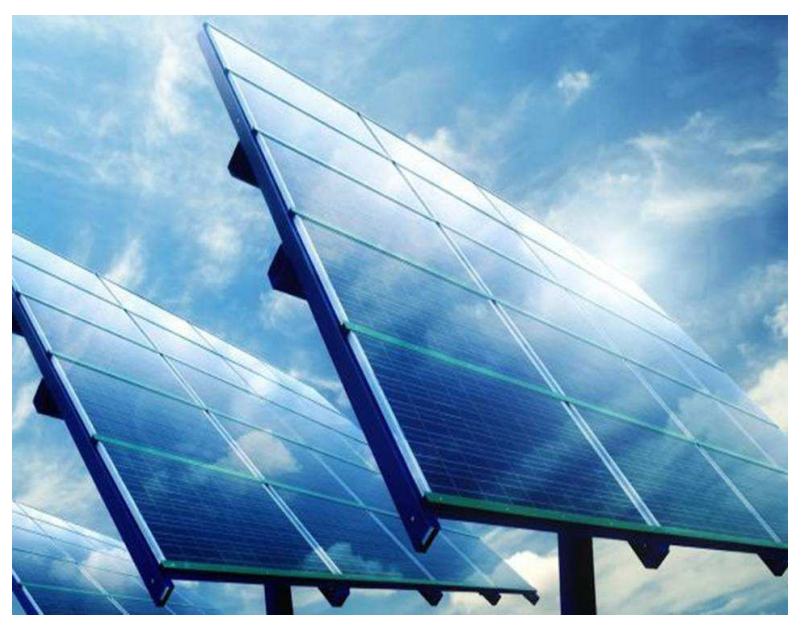

Fig. 1: The best panels which are also resistant to hail

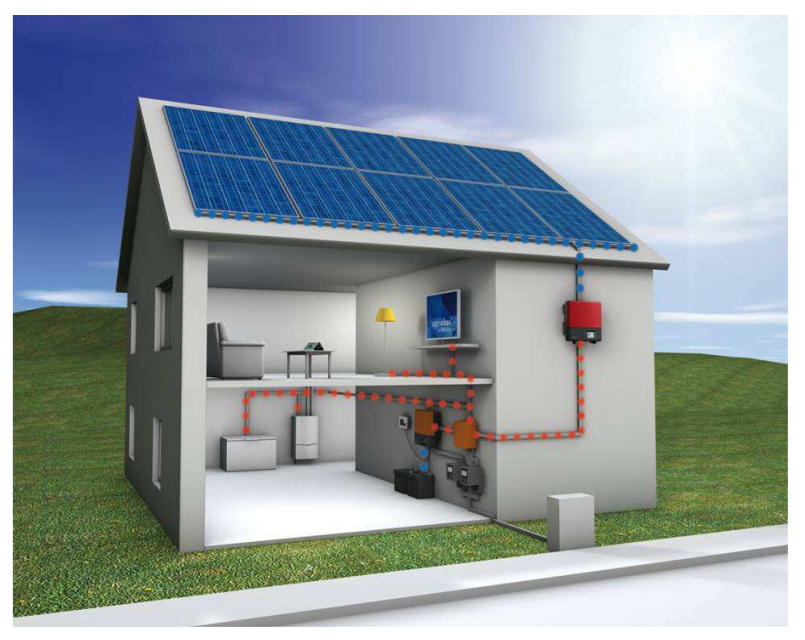

Fig. 2: The case for roofs of houses/blocks, adjacent buildings (covered parking lots, etc.) or land on which solar-thermal collectors can be located

Wind power applications involve electricity generation, with wind turbines operating in parallel to network or utility systems, in remote locations, in parallel with fossil-fueled engines (hybrid systems). The gain resulting from the wind energy exploitation consists both in the low consumption of fossil fuels as well as the reduction of the overall costs of generating electricity. Electric utilities have the flexibility to accept a contribution of about $20 \%$ of wind power systems. Combined Eolian-diesel systems can offer fuel savings of over $50 \%$.

Wind power generation is a fairly new industry $(20$ years ago in Europe, wind turbines had not yet reached commercial maturity). In some countries, wind energy is already competing with fossil fuel and nuclear energy, even without considering the benefits of wind energy for the environment. 


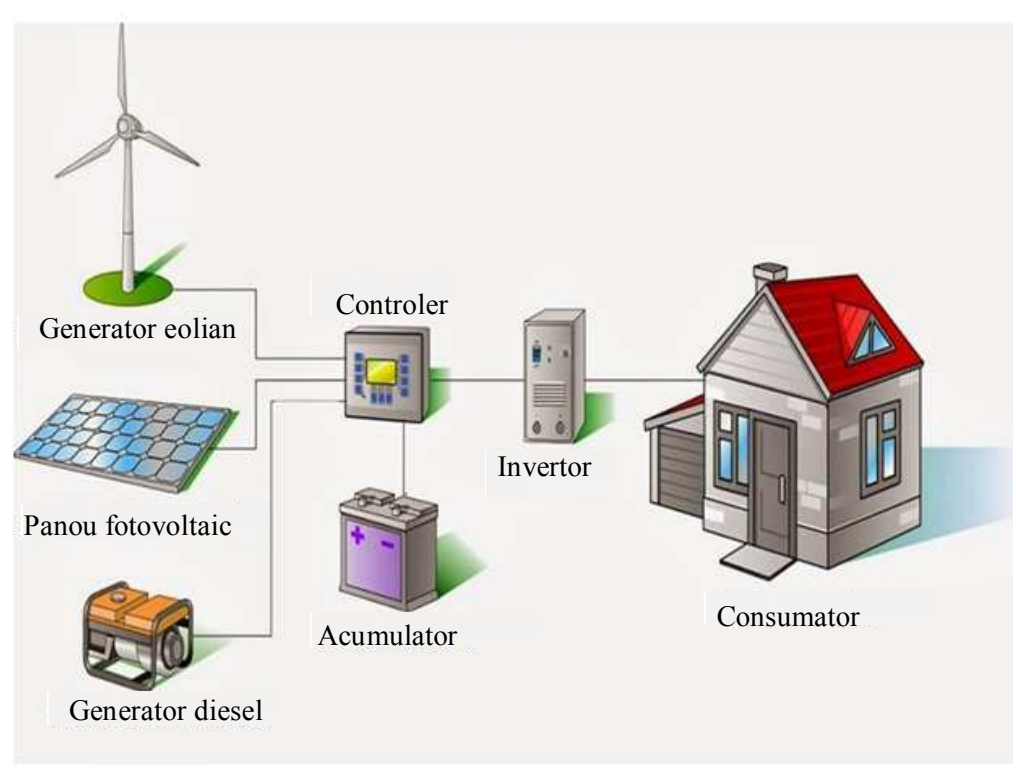

Fig. 3: A system for producing, distributing and maintaining renewable energies for a house, cottage, motel, hospital, even located in isolated places, where the power grid does not reach

When estimating the cost of electricity produced in conventional power plants, their influence on the environment (acid rain, effects of climate change, etc.) is usually not taken into account. Wind energy production continues to improve by reducing costs and increasing efficiency.

The cost of wind energy is between 5-8 cents per $\mathrm{kWh}$ and is expected to fall to 4 cents per $\mathrm{kWh}$ in the near future. Maintenance of wind energy projects is simple and inexpensive. Amounts of money paid to farmers for land renting provide additional income to rural communities. Local companies that carry out the construction of wind farms provide short-term local jobs while long-term jobs are created for maintenance work. Wind energy is a rapidly growing industry in the world.

An indispensable requirement for the use of wind to produce energy is a constant flow of strong wind. The maximum power Wind Turbines (WTS) are designed to generate is called "rated power" and the wind speed at which nominal power is reached is "wind speed at rated power". This is chosen to suit the wind speed in the field and is generally about 1.5 times the average wind speed in the ground. One way to classify wind speed is the Beaufort scale that provides a description of the wind characteristics. It was originally designed for sailors and described the state of the sea, but was later modified to include wind effects in the field.

The power produced by the wind turbine increases from zero, below the starting wind speed (usually around $4 \mathrm{~m} / \mathrm{s}$, but again, depending on the location) to the maximum at wind speed at rated power. Above the wind speed at rated power, the wind turbine continues to produce the same rated power, but at lower output until it stops, when the wind speed becomes dangerously high, ie over 25 to $30 \mathrm{~m} / \mathrm{s}$ (vigorous storm). This is the shut off speed of the wind turbine. Exact specifications for identifying the energy produced by a wind turbine depend on the wind speed distribution during the year in the field.

Air currents can be used to train wind turbines. Modern wind turbines produce a power of between 600 KW and $5 \mathrm{MW}$, the most used being the 1.5-3 MW output power, being more simple and constructive and more suitable for commercial use. The output power of a typical wind turbine is dependent on the wind speed at the third power so that wind speed increases, the power generated by the turbine increases with the wind speed cube, the increase being spectacular. The world's technical potential for wind power can provide five times more energy than it is consumed now.

In the strategy for capitalizing on renewable energy sources, the declared wind potential is 14,000 MW (installed power), which can provide an amount of energy of about 23,000 $\mathrm{GWh} /$ year. These values represent an estimate of the theoretical potential and must be reproduced in correlation with the possibilities of technical and economic exploitation. Starting from the theoretical wind potential, what interests the energy development forecasts is the potential for practical use in wind applications, which is much smaller than the theoretical potential, depending on the possibilities of land use and the conditions on the energy market. That is why the economically profitable wind potential can be appreciated only in the medium term, based on the technological and economic data known today and considered as valid in the medium term. 
Under ideal conditions, the theoretical maximum of cp is $16 / 27=0.593$ (known as the Betz limit) or, in other words, a wind turbine can theoretically extract $59.3 \%$ of the airflow energy. Under real conditions, the power factor does not reach more than $50 \%$, as it includes all wind turbine wind turbine losses. In most of today's technical publications, the $\mathrm{cp}$ value covers all losses and represents the product $\mathrm{cp} * \mathrm{~h}$. The power output and the extraction potential differ depending on the power coefficient and the turbine efficiency.

If $\mathrm{cp}$ reaches the theoretical maximum, the wind speed immediately behind the rotor - v2 is only $1 / 3$ of the speed in front of the rotor v1. Therefore wind turbines located in a wind farm produce less energy as a result of the reduction in wind speed caused by the wind turbines in front of them. Increasing the distance between wind turbines can reduce energy loss as wind flow will accelerate again. A correctly designed wind farm may therefore have less than $10 \%$ losses due to mutual interference effects (Fig. 4).

Average annual power will vary from land to land. In high wind speeds, more energy will be obtained. This highlights the importance of strong winds and hence the implications of the wind climate on economic issues related to wind energy production.

Blasts are responsible for mixing air and their action can be considered in a similar way to molecular diffusion. As the vortex passes through the measuring point, the wind speed takes the value of that whirlpool for a period of time proportional to the magnitude of the whirlpool; this is a "gust". In most cases, load variation is not significant. However, if the vortex scale is of the same magnitude as the scale of a component of the turbine, then the variation in load may affect the overall component. A gust of $3 \mathrm{sec}$ corresponds to a whirl of about $20 \mathrm{~m}$ (e.g., similar to the length of a rotor blade), while a $15 \mathrm{sec}$ burst corresponds to a $50 \mathrm{~m}$ swirl.

To calculate the maximum possible load for a turbine or its components over the lifetime of the turbine, the highest burst value is used for a relevant period of time. This is formulated as the maximum wind speed and gust speed over a 50 -year period. Of course, wind speed can be exceeded during this period, the sizing reserve will allow for some overtaking. Calculation of stresses is particularly important for flexible structures, such as turbines, which are more susceptible to wind-induced damage than rigid structures such as buildings (Fig. 5).

A wind turbine can be placed almost anywhere in a sufficiently open terrain. Nevertheless, a wind farm is a commercial project and therefore it is necessary to try to optimize its profitability. This is important not only for the profitability during the lifetime of the exploitation but also for the mobilization of capital in the initial phase of project development. For economical planning of investments in wind energy, it is necessary to know as safely as possible the prevailing wind conditions in the area of interest.

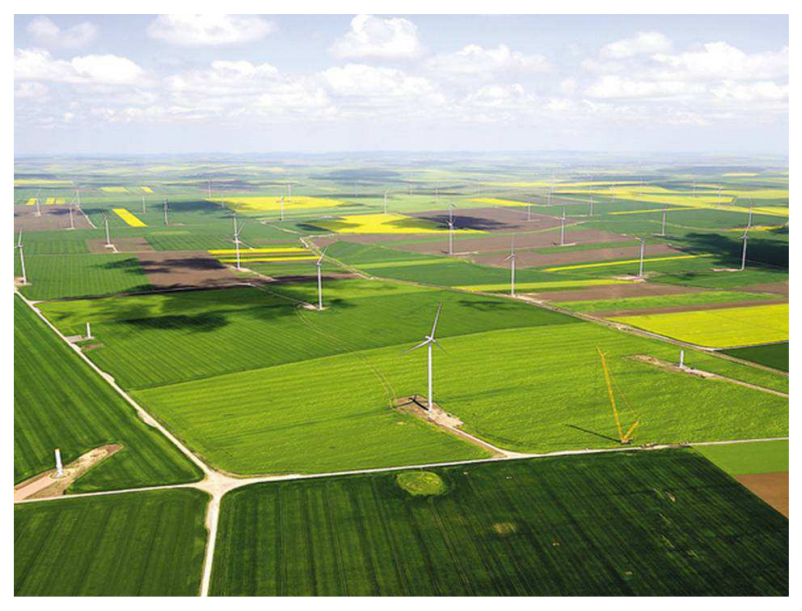

Fig. 4: Location of modern wind farms on a field

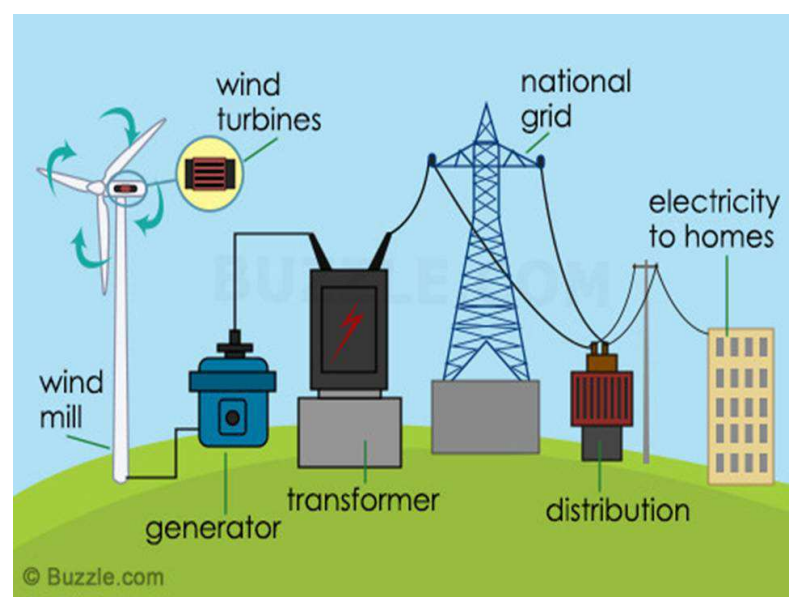

Fig. 5: Wind power coupling system to the national grid

Due to lack of time and financial reasons, long-term measurement periods are often avoided. As a substitute, mathematical methods can be used to predict wind speeds at each location. Wind conditions and energy production resulting from the calculation can serve as the basis for economic calculations. In addition, simulation of wind conditions can be used to correlate wind measurements at a particular location with wind conditions in neighboring locations in order to determine the wind regime for a whole area.

Since wind speed can vary significantly over short distances, for example several hundred $\mathrm{m}$, wind turbine location assessment procedures generally take into account all regional parameters that are likely to influence wind conditions.

Such parameters are:

- Obstacles in the immediate vicinity

- Topography of the environment in the measure region, which is characterized by vegetation, land use and buildings (description of the roughness of the soil) 
- Horoscopes, such as hills, may cause airflow acceleration or deceleration effects

For the calculation of the annual average power density in the field, a more accurate estimate of the average annual wind speed is required. Then, information on the wind speed distribution over time is needed. To obtain these trusted data, data sets that cover several years are needed, but usually these data are estimated using appropriate models from shorter-date data sets. After that, it is possible to determine the potential energy produced in relation to the performance of the wind turbine.

The most widespread procedure for long-term prediction of wind speed and energy efficiency in a land is the WAsP Wind Atlas.

The model quantifies the wind potential at different heights of the rotor shaft above ground for different locations, taking into account the distribution of wind speed (in time and space) at meteorological stations (measurement points).

The reference station could be up to $50 \mathrm{~km}$ away from the site. The projected energy output for this location can be calculated in relation to the power curve associated with the wind turbine (wind power). A key element of the WASP model is that it uses polar coordinates for the origin of the location of interest.

The WAsP incorporates both physical atmospheric models and statistical descriptions of the wind climate.

The physical models used include:

- The similarity in the surface layer - considering the logarithmic law

- The Geostrophic Wind Law

- Stability corrections-to allow for variation from neutral stability

- Change of roughness-to allow changes in land use throughout the area

- Shelter model-modeling the effect of an obstacle on wind flow

- Orographic model-modeling the effect of accelerating the wind speed in the field

The wind regime is described statistically by a Weibull distribution derived from the reference data. The Weibull distribution is designed to best fit the high wind speeds.

Depending on the complexity of the examined regions, different procedures are used to determine the wind conditions. In addition to the WAsP model mentioned above, there are other procedures such as mesoscales models.

Such measurements, usually performed over a period of one year, may be related to neighboring areas or may be extrapolated to the height of the rotor axis of certain types of turbines using the flow simulation described above.

Evaluating wind resources at a location ideally asks for data series for as long as possible at the location of the turbines. In addition, it is useful to understand the turbulence in the field and the rotor axis for the design of wind turbines. To do this, a quick time sample and spatial distribution of measurement points is required. In practice, time and expenses often exclude such a thorough investigation. Imitations are provided in the section on meteorology and wind structure.

Wind velocity measurements are the most critical measurements for wind resource valuation, performance determination and energy production. In economic terms, uncertainties are transformed directly into financial risk. There is no other branch in which the uncertainty of the measurements is as important as in wind energy. Due to the lack of experience, a lot of wind speed measurements have inaccurately high uncertainties, as best practices in the anemometer selection standards, anemometer calibration, anemometer fitting and measurement field selection have not been applied.

Investigations have shown that certain anemometers are highly susceptible to vertical air flow, which, under real conditions, even appear in open ground due to turbulence. In complex terrain these effects are of great importance and lead to over or underestimation of real wind conditions. Only a few types of anemometers can avoid these effects.

A representative positioning of the measuring point within the wind farm shall be chosen. For large power plants in complex terrain, 2 or 3 representative positions should be chosen for the installation of the pillar. At least one of the measurements must be made at the height of the rotor shaft of the future turbine to be installed, since extrapolation from a smaller height to the height of the rotor shaft gives rise to additional uncertainties. If one of the measuring posts is positioned close to the wind farm area, it can be used as the reference wind speed measuring pole during the boiler operation and for determining the wind energy performance by sectors.

Measurement of wind speed and direction are obviously necessary, but also other parameters, particularly air pressure and temperature. The equipment used for these measurements must be robust and safe, since it will generally be left unattended for long periods of time.

Average wind speed is usually collected using cup anemometers because they are safe and cheaper. These anemometers often have better response characteristics than those used at weather observation centers. Wind direction is measured with a girue. Giruetes are usually wound potentiometers. Giruge will be affected by the shade of the pillar and is often oriented so that the pillar is in the least likely wind direction. If data about the vertical flow of air is required, three-dimensional data is useful. These are obtained if lesser robust propeller anemometers are used, or sonic anemometers, which are more expensive.

These anemometers indicate information about both speed and wind direction. The data should be taken at a high frequency, possibly $20 \mathrm{~Hz}$. 
It is important that data transmission and storage is secure. For this purpose, the logger must be carefully isolated from atmospheric conditions, especially rain. Many experiments have suffered significant data loss due to various problems, including water infiltration or loss of electricity. The most promising locations for wind farms are usually in hostile environments, but a host of trusted data loggers are available on the market.

It is possible to collect data remotely and download data via a telephone line. The advantage is that data can be monitored on a regular basis and any other problems that occur with the tools can be resolved quickly. For the development of a wind energy project it is essential to carefully plan the data collection step.

Daily weather information is usually available free of charge from weather services. However, for statistical data and consultancy services fees are charged.

In South Europe, the wind regime is dominated by seasonal winds. Winter cold weather is associated with the northern and northest winds. These variations can be seen in station records for wind speed and temperature.

Certain studies suggest that a minimum of 8 months of data is required for the adequate estimation of wind resources. Other researchers have suggested that winter wind is the most important because it coincides with peak demand for electricity. The data can then be sorted into ranges or "boxes" for wind speed or wind direction, either as a whole. The number of measurements in each box is then counted and the sorted data is plotted as a percentage of the total number of readings to indicate the frequency distribution.

From these data it is possible to calculate the average wind speed and wind speed most likely. It is possible to obtain the distribution of the wind power density (proportional to the cubic wind speed). Data may also be presented as the probability of a higher wind velocity than another given value, usually zero, $u>0$. These data can be represented by two parameters from the Weibull distribution, the $\mathrm{k}$ and $\mathrm{C}$ parameters resulting from the use of certain techniques such as the moment method, the least squares method and many others. The two parameters of the Weibull distribution match for many wind data with acceptable accuracy.

The data collected are representative, for example, that the year is not particularly windy or calm. To be sure, data is needed for about 10 years. Obviously this is not practical for a location. However, it is possible to compare the wind data from the location with those of a nearby weather station and apply a MCP-type methodology to increase the data set actually measured at 10 years.

There are a number of available MCP methods, such as:

1. Calculate the Weibull parameters from the location of interest and the reference location and correlate them over the measurement period and then apply the correction for the rest of the reference data
2. Calculation of the correction factor (coefficient) for the wind speed between the location of interest and the reference point, during the measurements and on each step of the wind direction

3. Correlate measured data with reference data by determining a continuous function between the two for all data over the measurement period and applying it for the rest of the reference data

Once the wind distribution probability density is established, the power curve of a turbine can be correlated with wind data to determine the turbine power density density. The data can of course apply to different types and configurations of turbines for optimizing results.

The annual energy output of a wind turbine is the most important economic factor. Uncertainties in determining the annual speed and power curve contribute to the overall uncertainty of predicted annual energy production and lead to a high financial risk.

Annual energy production can be estimated by the following two methods:

- Wind velocity histogram and power curve

- Theoretical wind distribution and power curve

In addition to the wind regime, there are several factors that must be considered at the final choice of the optimal location for the installation of the wind farm. Mostly these include:

- Access to the electricity network

- Access road

- Local effects on the environment, including landscape damage

- Approaching housing

- Noise effects

- Interference with radio and TV signals

The locations of wind farms and the associated weather conditions have made engineers face many challenges to meet the design requirements of the plants and installed systems. Poor access to the site may prevent large and heavy components from being delivered, the rocky terrain makes it difficult to install as well as the electrical grounding system and rain and fog can lead to water infiltration in the cable connections.

The construction and operation of a wind power plant require the use of heavy equipment for the preparation of the land, the transport of construction materials and the components of the project, as well as for the lifting of turbines, electric poles and towers. Thus, there could be a potential risk that wind projects will affect rural roads designed for low traffic or light vehicles. Existing rooftops should be rebuilt or reinforced to withstand additional loads without degrading and the frequency of planned maintenance for these roads could increase. 
It is generally recommended:

- Construction of small roads and use of maintenance techniques to reduce a temporary or permanent loss of land

- $\quad$ Restricting vehicles for existing access roads

- Limiting the number of new access roads, the width of new roads and avoiding or minimizing cuts or fillings

- Building new access roads that follow the existing contours to the greatest possible extent

Wind turbines are typically located in rural or mountain areas, where the connection to the nearest substation may be weak and local demand for electricity may be much lower than the capacity of the boiler plant. One way to define the "power" of the transmission network is the fault level, which is a measure of the current to flow when a network failure occurs. At the end of a long electric circuit, the fault level is much lower than in the center of an interconnected network, for example in a city or industrial center.

At a low defect level, the impact of wind turbines may be large enough to disturb other local consumers. For this reason, in some cases, it is necessary to strengthen the network, or to connect the network to a higher voltage or to a more remote area where the network is strengthened. This will increase costs.

In rural or mountainous areas, it is preferable that the closest point of the power grid be to an over ground line rather than underground. Here you can find a number of electric poles or pillars that will help FPE engineers locate the land of interest on their system map and then be able to define the voltage of the power line. Any twowire airline is in one-phase system and normally requires reinforcement if generators are to be installed.

The technically-economical design of the electrical collection system for a power plant and its connection to the power grid is a process of optimizing several parameters and requires extensive experience from the designer/engineer as well as the availability of mild calculation systems to easily find the best solution.

Typically, the overall appearance of the wind farm is based on optimizing the park's production with regard to the location of individual turbines and their accessibility - That is infrastructure. The contribution of the short-circuit in the network is an important parameter, depending on the availability and evaluation of electrical equipment Transformers, cables, main ring units, switches, etc. Choose a solution that meets the basic electrical design requirements and check it by defective current calculations.

The loss calculation is based on the wind farm production profile, calculated from the parameters that describe the wind - the parameters of the Weibull distribution - and the power curve of the wind turbines concerned.
Wind power plants offer a number of important advances compared to conventional coal, oil or natural gas plants; i.e., do not use fuel, do not emit pollutants, greenhouse gases, or toxic waste and do not consume water or other rare resources. However, wind power plants can raise environmental and community concerns. For example, they generate noise and can be visually intrusive for residents living near them. They can also disrupt wild habitats and cause the wounding or death of birds.

The construction and operation of a wind power plant involve many of the construction and operation activities of a conventional power plant, including road construction, land clearing, trafficking in large-scale machinery and construction of connection and transport lines. In addition, wind projects raise community-related issues, particularly with regard to visual impact and noise.

Unlike most power plants, wind power projects are more intrusive than terrain than intensive. For the production of a MW, the land needed for a wind energy project exceeds the land needed for most of the energy generation technologies. However, while wind facilities can expand on a large geographic area and have a wide range of influence, the physical footprint project covers a relatively small portion of this field. A wind power project of $50 \mathrm{MW}$, for example, can occupy a land of 1500 acres, but the actual land area occupied by wind power plants may be only three to five percent of the total area, leaving the rest available for other uses compatible.

Since wind energy production is limited to areas where weather conditions predict a relatively long season of strong and consistent wind resources, the development of wind projects worldwide has mainly occurred in rural and relatively open areas. These lands are often used for agriculture, grazing, recreation, open spaces, scenic areas, wildlife habitat, forest management and seasonal irrigation deposit. The development of wind energy is usually compatible with farm or grazing a site.

Developing wind projects may affect other uses in/or adjacent to a site (location), or in the surrounding area. Some recreational parks and uses that highlight wildlife values and wildlife conservation reserves - especially birds-can't be compatible with the development of nearby wind projects.

Wind turbines are extremely visible structures. Modern wind turbine towers measure from 30 to $50 \mathrm{~m}$ above ground without counting the rotor blades that can reach up to 40 feet in diameter. In addition, most of the times the turbines are arranged in dozens of paintings or more on visible hills and hills. If the visual impact of wind turbines generates complaints depends in part on where they are installed.

Whatever the location, action can be taken to reduce the number of complaints by building less impressive and more enjoyable turbines. For example, tubular towers are less offensive than lattice towers.

The vast majority of those affected by the noise produced by wind turbines live a few miles from high- 
powered wind power plants or a few hundred $\mathrm{m}$ from a small or individual turbine plant. Although the noise at these distances is not very high - a $300 \mathrm{~kW}$ turbine produces a noise level at $120 \mathrm{~m}$ less than that produced by a traffic light at $30 \mathrm{~m}$ away - it is still loud enough to hear in the rooms and can be significant at night when traffic and home noise are diminished.

When planning a wind project, attention should be paid to any noise that could be heard outside the nearby buildings. Inside, the noise level is probably lower even with the windows open. The noise that will be produced when the wind blows from the turbines to the houses, usually characterizes the impact of potential noise. It is then compared to the background noise already existing in the area without the wind farm functioning.

In recent years, the potential effects of wind projects on wildlife and natural spaces have been highlighted.

There may be problems in many locations, as some of the characteristics of a good location for wind farms are also attractive for birds. For example, mountain passes are frequently windy because they provide a channel for the wind to pass through the mountain; for the same reason, they are often the preferred routes for migratory birds.

An important aspect is the significance of deaths and bodily injuries to the local bird population. Ideally, birds should not be killed, but this is not practicable in many cases.

Some studies have shown that birds and other animals tend to avoid nesting or hunting in the immediate vicinity of wind turbines. In addition, activities such as road construction and tree deforestation can destroy habitats and allow the introduction of unwanted species. The problem is aggravated by the fact that the best turbine locations are located isolated in areas that host different plant and animal species.

During the development of a wind project, locations with archaeological or cultural resonance must be protected and avoided. The potential impact area of a wind project may range from a few dozen meters up to a kilometer or more. That is why in the set of approvals required for a wind project is usually required the opinion of the authorities in the field of culture and archaeological heritage. During the installation of the wind infrastructure, archaeological monitoring is carried out by the competent authorities for sites that may contain archaeological vestiges.

In general, in the site selection process, a site plan is required to certify the conditions for fitting a wind farm. Such plans shall establish conditions and criteria such as:

- Wind turbine size, including maximum rotor size, minimum and maximum height, tower height, etc

- Installation and design including tower and rotor, utilities notification, warning signs and tower access
- Set-up, including the withdrawal distance of the boiler from neighboring facilities, roads, other wind power plants, aesthetic design (such as a tubular or lattice tower) and proximity to the power line

- Noise and radio and TV interference regulations

- Other regulations, including insurance, public access to wind installations and decommissioning requirements

\section{The Micro-Hydropower Potential}

Hydroelectric power comes from the action of moving water. It can be seen as a form of solar energy because the sun feeds the water circuit in nature. Within this circuit, the water from the atmosphere reaches the surface of the earth in the form of precipitation. Part of it evaporates, but much of it penetrates the soil or becomes flowing water to the surface. Rainwater and melted snow finally end up in ponds, lakes, reservoirs or oceans where evaporation takes place permanently.

Water resources due to inland rivers are estimated at about 42 billion cubic meters per year, but under unchecked storage, it can only account for about 19 million cubic meters per year due to fluctuations in river flows.

Low-power hydropower plants are a major contributor of renewable electricity at European and world level. Worldwide, it is estimated that there is an installed capacity of 47,000 MW, with a potential technical and economic - close to 180,000 MW.

Low-Power Hydropower Plants (HMP) are powered by natural water flow, i.e., it does not involve large-scale water capture and therefore does not require the construction of large dams and reservoirs, although they help where they exist and can be used easily. There is no international definition of the HMP and the upper limit varies between 2.5 and $25 \mathrm{MW}$ depending on the country, but the $10 \mathrm{MW}$ value is generally accepted and promoted by European Association of Low Power Hydro Power Plants (ESHA).

Low power plants are one of the most reliable and costeffective technologies for producing clean electricity.

In particular, the key advantages of HMPs to windbased, wave-based or solar power plants are:

- High efficiency (70-90\%), by far the best of all energy technologies

- A high capacity factor (usually> 50\%), compared to $10 \%$ for solar energy and $30 \%$ for wind power

- High predictability, depending on yearly rainfall patterns

- Low rate of variability; The energy produced varies only gradually from day to day (not from one minute to the next)

- Good correlation with demand (eg output is maximum in winter)

- It is a sustainable and solid technology; Systems can be designed to work for over 50 years 
HMPs are also environmentally friendly. Most of the time, they work on the natural course of water. Therefore, this type of water-based installation does not have the same negative environmental effects as large hydropower plants.

Small hydropower plants can be located either in mountainous areas where rivers are fast or in low-lying areas with large rivers. The four most common types of micro-power plants are presented below.

For large and medium fall schemes, channel and duct combinations are used. If the terrain is injured, the construction of the canal is difficult and then only the forced duct that can sometimes be buried is used. In the barrage arrangements the turbines are placed in or in the immediate vicinity of the dam, so that there is almost no need for the channel or the pipeline.

Another option of placing the microturbines is to use the flows from the water treatment plants.

The objective of a hydroelectric system is to convert the potential energy of the volume of water flowing from a certain height into electricity at the bottom end of the system where the power plant is located. The water level difference, known as "fall", is essential for the production of hydroelectricity; The simple rapid flow of water does not contain enough energy to produce significant electrical energy than on a very large scale such as coastal submarine currents. That is why two indicators are needed: $\mathrm{Q}$ water flow and $\mathrm{H}$ dropping. It is generally better to have a larger drop than a higher flow, because smaller equipment can be used.

Grossfall $(\mathrm{H})$ is the maximum vertical distance between upstream and downstream water levels. The actual fall seen at the turbine will be somewhat lower than the gross fall, due to the loss of water in and out of the system. This low fall is called the Net Fall.

Flow rate $(\mathrm{Q})$ is the volume of water passing into the unit of time, measured in $\mathrm{m}^{3} / \mathrm{s}$. For small systems, the flow rate can also be expressed in liters/second, where $1000 \mathrm{l} / \mathrm{s}=1 \mathrm{~m}^{3} / \mathrm{sec}$. Depending on the fall, hydroelectric plants can be classified into three categories:

- Large drop: Over $100 \mathrm{~m}$

- Average fall: $30-100 \mathrm{~m}$

- $\quad$ Reduced fall: 2-30 m

These categories are not strict, but are only a possible ranking system for locations.

Hydroelectric installations can also be defined as:

- Installations on the water wire

- Installations with a power plant located at the base of a dam

- Integrated systems on a channel or in a water supply pipe

Generally, large-scale locations are less expensive to develop than small-fall ones, because for the same level of energy produced, the flow required by the turbine will be lower than hydro-technical constructions. For a river with a relatively high slope in a sector of its course, the level difference can be used by conducting part or all of the course and returning it to the river bed after passing through the turbine. The water can be brought directly from the source to the turbine via a pressure pipe.

Hydroelectric turbines convert water pressure into mechanical power to the shaft, which can be used to drive an electric generator or other equipment. Available electricity is directly proportional to the fall and flow rate.

The best turbines can have hydraulic efficiency in the order of $80-90 \%$ (higher than any other driving force), although it decreases with size.

The main component of a small hydropower plant is the hydraulic turbine. All of these turbines convert the falling water energy into kinetic rotation shaft energy, but confusion often arises as to which type of turbine should be used depending on the circumstances. The choice of the turbine depends on the location characteristics, especially the drop and flow, plus the desired generator speed and if the turbine has to operate under low flow conditions.

There are two main types of turbines, called "impulse" and "reactive".

The impulse turbine converts the potential energy of the water into kinetic energy through a jet that comes out of a nozzle and is projected onto the rotor cups or blades.

The reaction turbine uses pressure and water speed to create energy. The rotor is completely immersed and the pressure and speed drop from intake to exhaust. By contrast, the rotor of a pulse turbine operates in air, driven by a jet (or jets) of water.

There are 3 main types of impetus turbines: Pelton, Turbo and Cross Flow (or Banki). The main 2 types of reaction turbines are helical (Kaplan) and Francis.

Most existing turbines can be grouped into three categories:

- Kaplan and helical turbines

- Turbine Francis

- Pelton turbines and other impulse turbines

Kaplan and propeller turbines are axial flow turbines, generally used for small falls (typically less than $16 \mathrm{~m}$ ). The Kaplan turbine has adjustable blades and may or may not have an adjustable stator head unit. If both the rotor blades and the steering gear are adjustable, we are dealing with a 'double-tuned' turbine. If the directing device is fixed, we are dealing with a 'simple tuned' turbine. In the conventional version, the Kaplan turbine has a spiral chamber (either steel or reinforced concrete); the flow enters radially inward and makes a straight angle before entering the rotor in the axial direction. If the rotor has fixed blades, the turbine is called a propeller turbine.

Propeller turbines may have mobile or fixed devices. Turbines with nipples are used only if flow and fall are practically constant. 
Bulb and tubular turbines are derived from the Kaplan and helical variants, where the flow enters and exits with minor directional changes. In the Bulb turbine, the multiplier and the generator are located in a submerged capsule. The tubular turbines allow several arrangements, namely: Straight-angle transmission, Straflo turbines with S-ducts, belt drive generators, etc. Versions with straightangle transmission are very attractive, but they are only manufactured to a power of $2 \mathrm{MW}$.

Francis turbines are radial-flow turbine engines with fixed rotor blades and mobile guides used for mid-fall. The rotor is made up of cups with complex profiles. A Francis turbine typically includes a spiral cast iron or steel chamber to distribute water throughout the perimeter of the rotor and a series of guide elements to adjust the flow of water into the rotor.

Pelton turbines are single or multiple jet turbines, each jet being designed with a needle nozzle to control the flow. They are used for medium and large falls. The nozzle axes are on the rotor plane.

The cross-flow turbine, sometimes called the Ossberger turbine, after a company that has been manufacturing it for over 50 years, or the Michell turbine is used for a wide range of falls, overlapping with Kaplan, Francis and Pelton turbine applications. This type is very suitable for a high-flow and low drop stream.

Turbo can operate under a fall ranging from $30-300 \mathrm{~m}$. Like the Pelton turbine, it is a pulsating turbine, but the blades have a different shape and the water jets hit the plane of the rotor at an angle of $20^{\circ}$. The water enters the rotor through one side of it and goes out through the other. The high turbo turbine speed due to its smaller diameter than other models makes it more likely to directly engage the turbine and generator. A turbine of this type may be suitable for average falls where a Francis turbine could also be used. But, unlike Pelton, the water passing through the rotor produces an axial force that requires the installation of a transmission shaft on the shaft.

The type, geometry and dimensions of the turbine will be fundamentally conditioned by the following criteria:

- The net fall

- Turbine flow ranges

- Rotation speed

- Cavity problems

- Cost

The efficiency of a turbine is defined as the ratio between the power delivered by the turbine (mechanical power transmitted to the axle) and the absorbed power (the hydraulic power equivalent to the flow measured under the net fall). To estimate overall efficiency, the efficiency of the turbine must be multiplied by the efficiency of the speed multiplier (if used) and the alternator.
For different types of turbines, efficiency drops rapidly below a certain percentage of nominal flow. A turbine is designed to operate close to the maximum efficiency point, typically $80 \%$ of the turbine's maximum flow and when the flow deviates from this value, the hydraulic efficiency of the turbine decreases.

The flow rate range and thus the generated energy, varies if:

- The system must provide power to a small network

- The system was designed to connect to an extended distribution network

In the first case, it is necessary to select a flow that allows the production of energy almost all year round. In the second case, the nominal flow should be selected so that the net profit from the sale of electricity is maximum.

The control panel is the equipment that monitors the operation of the hydropower system. The main functions of the control panel are:

- Turning the turbine on and off

- Synchronize the generator with the local network

- Monitoring the upstream water level and ensuring that it is kept above the minimum

- Operation of the flow control valve to the turbine to coordinate with the availability of water

- Detection of faults and activation of warnings or stop sequences

A filter grating is a grate used to filter waste water. This is a useful equipment to prevent the accumulation of waste and other undesirable elements in watercourses, rivers and lakes. The basic scheme of all filter hatches is similar, but internal, external and turbine grates serve different needs. Grates can also be produced from different materials.

A simple filtration grate for a watercourse can be made of any type of barbecue material that allows water to pass but retains much of the waste. A filter of this type is usually made of the same material as whipped cream, i.e., metal or plastic.

Depending on the water course and the level of pollutants passing, the grate filter often requires regular cleaning to avoid blocking the water course. The grill is an obstacle and leads to a slight reduction of the frame. Therefore, the distance between the bars must be the maximum that allows for the collection of large enough waste for a turbine damage. The turbine manufacturer will recommend the correct dimensions.

Also, the flow rate of water near the filter grating should be relatively low, preferably below $0.3 \mathrm{~m} / \mathrm{s}$ and not more than $0.5 \mathrm{~m} / \mathrm{s}$.

Manual cleaning is only feasible for small installations or locations that have permanent staff for other reasons. 
There is now a range of automatic cleaning equipment available to remove waste collected by filter gratings.

The most common types are:

- Robotic Bracket: These are of several types, usually with one or more rakes operated by a hydraulic piston. Some models require only one rake that can sweep along the grill; in other cases two or more rakes can work side by side. These systems are usually very robust, partly because they keep their drives out of the water. The main disadvantage is the visual presence of the equipment and the higher security risk raised by the unattended operation of the equipment

- Rake-chain cleaning system, where a bar is moved up on the grill by a chain transmission at each end. The bar stores the collected waste in a channel that runs across the length of the filter grating. The channel can be washed with water (pumped if needed), which trains the waste to a lateral spillway

- Grip-lifting system is a robust alternative to the robotic rake. A pair of 'jaws' scrabble the grate and raises the materials directly into a dump

- Candash filter systems, which are only usable for medium and high drop systems, do not require raking because they use the Coanda effect to filter and remove waste and alluviums, allowing only clean water access to the intake system. Stainless steel cables precisely positioned and arranged horizontally at small distances are embedded in a specially shaped filter that is installed at the downstream end of the inlet. Clean water is collected in a room under the filters, directly connected to the forced duct of the turbine

On rivers where there are concerns about fish safety, more stringent filtering regulations are usually applied to ensure that the fish are prevented from reaching the turbine intake and will be diverted to deviation. The specific fish filtering measures are set according to the sensitivity of the location.

Several innovative fish exclusion methods are tested in the intake areas, avoiding the use of a physical filter. These include the use of electricity, bubble curtains and sounds to guide the fish. These methods offer significant advantages for the operator, avoiding obstruction of the water flow.

Historical data of flows corresponding to a fixed location are considered for the estimation of water resources, so designers use this information. Ministries dealing with Environment, Hydrology, Energy and/or Environmental Agencies (national/regional/local) or other similar organizations are usually the source of flow measurements for the most significant rivers and watercourses in European countries. The data can be used to assess the flow rate of the watercourse at the proposed location, as long as it adapts to the ratio of the proposed location to the measurement site (downstream or upstream).
For the assessment of regional resources, satellite imagery is used to create the GIS database for source identification, site selection, environmental planning, Digital Terrain Modeling (DTM) and transmission line network and location classification. In general, these measurements for large-scale resource assessment are made by a team of GIS, hydrologists, hydropower experts, etc.

Geographic Information Systems (GIS) are computerized information systems used for digital representation and analysis of geographic features present on the earth's surface. The methodology for assessing the hydropower potential of a region can be done using both methods.

Remote sensing technology is an effective tool for identifying suitable locations for new hydropower projects, especially in inaccessible areas with high hydrological potential. Data from infrared remote sensing $(0.8 \mu \mathrm{m}-1.1 \mu \mathrm{m})$ clearly provides the contrast between water and earth and is therefore best suited for mapping perennial water courses.

The only resource required for a low-power hydroelectric plant is running water available at a certain angle. Planning a HMP starts with the most accurate estimate of the fall and flow available at the proposed location. There are several methods for measuring the fall available. Some methods are more suited for lowdrop locations, but they are too complicated and inaccurate for big falls. It is recommended to always take more fall measurements at each location.

The purpose of a hydrological study is to predict variation in flow over the year. Because the flow varies from day to day, a single measurement is of little use. In the absence of a hydrological analysis, a long-term measurement system can be installed. Such a system is often used to confirm the hydrological approach and is also the most reliable method of determining the actual flow rate at that location. Individual measurements are useful for verifying the hydrological predictions by sampling.

The flow measurement techniques are:

- the method to the bucket

- $\quad$ stage control method

- the salt method

- the bucket method

- the float method

- measuring the current

The theoretical potential for small hydropower plants and represents input data for calculations designed to estimate the technologically and economically exploitable potential.

The available potential is investigated by processing the above elements and after imposing some constraints related to:

- Legal and environmental aspects (land-use boundaries, minimum remaining flow) 
- General technical and economic problems (minimum flow, net fall, estimated energy production, forced column length/maximum distance from water inlet to the power plant)

To estimate the technological potential, the system then simulates the choice and operation of hypothetical turbines by using the following algorithms (for each hypothetical hydro power plant consisting of the available potential):

- Type of turbine and optimum installed capacity

- The energy produced

- Turbine utilization factor and available flow rate

The initial valuation of investment costs and financial feasibility calculations shall be calculated:

- Installation cost

- Operating and maintenance costs

- Cost of energy production (expressed in $€ / \mathrm{kWh}$ )

- Some Basic Indicators of Investment Profitability (IRR, NPV)

As a result, the system suggests some parts of the watercourse where low-power hydro power plants can be installed, with optimal power and financial efficiency.

A potential installation location of a HMP is defined by the location of the water intake and the location of the power plant construction next to the watercourse. The difference in height between these two locations is defined as the hydraulic (net) fall $\mathrm{h}$.

The assessment of a potential location should take into account the following features:

- Strong variation in water flow depending on climatic variations during the year, or differences between rich and poor hydrological years. This specific feature is quite intense when it comes to small water courses

- The type of hydro-turbines. As mentioned above, each type of turbine is adapted to certain values of the net fall (h) and the nominal flow rate Qr; Each has a different operating range, a different efficiency, the maximum value of which depends on the nominal turbine power, different sizes and costs

Low power plants have specific features different from large-scale ones, as they usually do not have large capacity upstream storage for financial reasons. It should be noted that large power plants (except those installed along the great rivers) have dams that form large accumulation basins. In this way, the natural flow of water is detached from the flow rate of the turbines, because the purpose of these large power plants is to cover the demand for energy at the peak points.
HMP, because of its low power, can't really contribute to the peak of energy demand and building up a build-up is therefore a disproportionate financial burden without benefits in relation to the investment. Therefore, an HMP, even using a water diversion, functions as a hydroelectric plant on the water line, which means that it is to exploit the natural flow as best as possible. This is why the HMP feasibility analyzes are made using the flow curve rather than the time series of the natural course because the HMP has no accumulation but only a limited basin whose volume provides good conditions for the water supply in Pipeline and which corresponds to the flow rate for several hours.

The analysis of the technically and financially exploitable hydropower potential is done separately for each watercourse. For each course, the system provides information about:

- Theoretical potential

- Potential available

- The technically and financially exploitable potential

\section{Theoretical Potential}

It is defined as the total potential energy available at selected nodes of the watercourse. The data used are: Nodes of the watercourse; - Yearly flow rate curve at at least one point of the watercourse; - Geographic data and the system calculates: - The annual flow curve of each water node, according to the law of equal surfaces (continuity); - difference in height between nodes; potential water potential for each branch of the watercourse.

Potential available

During the analysis of the potential of a watercourse, some availability filters are inserted, which effectively express some restrictions on the exploitation of water. Other non-energy uses of water form an important availability parameter for a river (irrigation, water supply, etc.). The system also provides some useful information on entities involved in water use rights for each part (segment) of the watercourse.

\section{Analysis on Each Watercourse}

In this section, a technical and economic analysis of all the hydropower plants that may be installed on a given watercourse is made. For each potential hydroelectric plant, the following parameters are estimated:

- Annual energy production

- Financial rating indices for the hydropower plant

The system continues with an assessment of possible hydro power plants based on their energy efficiency and financial feasibility and then provides the following information: 
- The most energy or financial power plants

- The most energy efficient or financially efficient plants that could be built simultaneously

Any developer must seek professional advice before allocating significant finance for the design and construction of a small hydropower system. Involvement of professionals in this type of project can go from preliminary site evaluation, a feasibility study to complete 'turnkey' service, dealing with every aspect of development. There are also several companies that are engaged in the rental, development and operation of locations and can provide a complete package of services and financing.

An experienced professional is able to decide if a location is worth investigating more closely, based on an initial visit and discussions with the developer and others. Preliminary investigations of this type typically require 2-3 days. At this stage, a minor investment could save much more expensive and other possible complications during the development process.

A feasibility study uses accurate data and carefully analyzes costs, moving the project from the original idea to a final project that will support project financing applications and the necessary licenses. That's why it is good to hire a professional to carry out the feasibility study and the detailed project.

In a feasibility study, we must find the following key elements:

1. Hydrological assessment. Typically, a hydrological assessment produces the flow curve. This will be based on long-term recordings of precipitation and/or flow data, together with geological data of the collection basin and soil types. This long-term information can be supported by short-term flow measurements. The study should also include an estimate of the required offset flow

2. System design. This includes the general description of the project, including the plan with the overall layout of the installation. The main aspects of the works must be covered in detail, such as: Construction works (inlet and inlet, intake duct, forced duct, turbine location, spillway, site access, construction details); Power generation equipment (turbine, gearbox, generator, control system); Connect to the network

3. System costs. They must include a detailed estimate of the capital costs associated with the project, broken down into:

- Construction costs

- Network connection costs

- Cost of electro-mechanical equipment

- Engineer fees and project manager fees

4. Estimation of energy production and annual income. It must use the source data (flows, hydraulic losses, net loss, turbine efficiency and calculation methods) and calculate the output of the system in terms of the maximum potential power (in $\mathrm{kW}$ ) and the annual average output $(\mathrm{kWh} /$ year) converted into annual revenue $€ /$ year)

\section{The Geothermal Energy Potential}

Geothermal energy is defined as the natural heat coming from within the Earth, captured for electricity, space heating or industrial steam. It is present anywhere beneath the earth's crust, although the highest temperature and therefore the most desirable resource, is concentrated in regions with active or young geologically active volcanoes.

The geothermal resource is clean, renewable, because the heat emanating from the Earth's interior is inexhaustible. The geothermal energy source is available $24 \mathrm{~h}$ a day, 365 days a year. By comparison, wind and solar energy sources are dependent on a number of factors, including daily and seasonal fluctuations and climate variations. For this reason, the energy produced from geothermal sources is, once captured, more secure than many other forms of electricity. Heat that continually springs from within the Earth is estimated to be equivalent to 42 million megawatts (Stacey and Loper, 1988). One megawatt can supply the energy needs of 1000 homes.

Geothermal energy originates from the thermal waters, which in turn extract their heat from the volcanic magma from the depths of the earth's crust. The Earth's thermal energy is therefore very large and is virtually inexhaustible, but it is very dispersed, very rarely concentrated and often too deep to be exploited industrially. Until now, the use of this energy has been limited to areas where geological conditions allow a transport medium (liquid or gaseous water) to "transfer" heat from hotspots from the depth to the surface, thus giving rise to geothermal resources.

The environmental impact of the use of geothermal energy is rather small and controllable. In fact, geothermal energy produces minimal atmospheric emissions. Emissions of nitrogen oxide, hydrogen sulphide, sulfur dioxide, ammonia, methane, dust and carbon dioxide emissions are extremely small, especially when compared to emissions from fossil fuels.

However, both water and condensed steam from geothermal power plants contain different chemical elements, including arsenic, mercury, lead, zinc, boron and sulfur, the toxicity of which obviously depends on their concentration. However, most of these elements remain in solution, in water that is reinjected into the same tank from which fermented water or steam was extracted. The most important parameter in the use of this type of energy is the temperature of the geothermal fluid, which determines the type of geothermal energy application. It can be used for heating or to generate electricity.

Going from the surface of the earth to the depth, it is noticed that the temperature increases progressively with 
the depth, with $3^{\circ} \mathrm{C}$ on average for every $100 \mathrm{~m}$ $\left(30^{\circ} \mathrm{C} / \mathrm{km}\right)$. It is called the geothermal gradient. For example, if the temperature after the first few meters below ground level, which on average corresponds to the average annual outdoor air temperature, is $15^{\circ} \mathrm{C}$ then it can reasonably be assumed that the first temperature will be $65-75^{\circ} \mathrm{C}$ at $2000 \mathrm{~m}$ Depth, $90-105^{\circ} \mathrm{C}$ at $3000 \mathrm{~m}$ and so on for the next few thousand meters.

Regions of interest for geothermal energy applications are those where the geothermal gradient is higher than normal. In some areas, either due to the volcanic activity of a recent geological age, or due to the cracked cracks of hot water at depths, the geological gradient is significantly higher than the average, so temperatures of $250-350^{\circ} \mathrm{C}$ are recorded at depths of 2000-4000 m.

A geothermal system consists of several main elements: a heat source, a reservoir, a carrier fluid that provides heat transport, a recharge area and a rock to seal the aquifer. The heat source may be a very high magmatic intrusion $\left(>600^{\circ} \mathrm{C}\right)$ that has reached relatively low depths $(5-10 \mathrm{~km})$ or, in some low temperature systems, the normal Earth's temperature, which, as explained earlier, increases with the depth.

The tank is a volume of permeable rocks from which the carrier fluid (water or steam) extracts heat. The reservoir is generally covered by either impermeable layers or rocks whose low permeability is due to the self-sealing phenomenon consisting in the deposition of minerals in the pores of the rocks. The tank is connected to a surface recharge area through which the meteoric waters can replace the fluids leaving the tank through springs or by extraction to boreholes. The geothermal fluid is water, in most cases meteoric, liquid or gaseous, depending on temperature and pressure. Water often carries along with chemicals and gases such as $\mathrm{CO} 2, \mathrm{H} 2 \mathrm{~S}$, etc. The mechanism underlying geothermal systems is generally governed by fluid convection. Convection occurs due to heating and thermal expansion of fluids in a gravitational field. The low density heated flame tends to rise and be replaced by a cooler, high density fluid coming from the edge of the system. Convection, by its nature, tends to increase the temperature at the top of the system, while the bottom temperature decreases. Frequently, a distinction is made between geothermal systems dominated by water and vapor-dominated systems. In water-dominated systems, liquid water is the continuous fluid phase controlling the pressure. Vapors may be present, generally as discrete bubbles. These geothermal systems, whose temperature may vary from $<125$ to $>225^{\circ} \mathrm{C}$, are the most widespread in the world. Depending on the temperature and pressure conditions, they can produce hot water, water-steam mixtures, wet steam, or, in some cases, dry steam. In vapordominated systems, liquid and vapor co-exist in the reservoir with continuous steam controlling the pressure. Geothermal systems of this type, of which the best known are Larderello in Italy and The Geysers in California, are quite rare and are high-temperature systems.

Generating electricity is the most important use of high pressure geothermal resources $\left(>150^{\circ} \mathrm{C}\right)$. Medium and low temperature resources are suitable for various applications. The classic Lindal Diagram (1973) shows the possible uses of geothermal fluids at different temperatures. Fluids at temperatures below $20^{\circ} \mathrm{C}$ are rarely used under very particular conditions, or in heat pump applications (Fig. 6-8), (DiPippo, 2004).

In the case of temperatures below $90^{\circ} \mathrm{C}$, geothermal waters can be used directly rather than for conversion to electricity.

The most common form of use is for space heating, agricultural applications, aquaculture and some industrial uses.

When the water temperature is below $40^{\circ} \mathrm{C}$, heat pumps are used to heat or cool the spaces. If groundwater is not available then heat pumps can be combined with heat exchangers with the ground.

A heat pump is a thermal machine which allows the extraction of heat from the basement and the aquifer at low depths (tens or hundreds of meters) at low temperatures and transferring it at higher temperatures to the medium to be heated. The advantage of heat pumps is related to the fact that for each unit of electricity consumed, approximately three units of heat in the form of heat, with the contribution of geothermal water, are obtained.

In the case of cooling, the heat is extracted from space and dissipated in the Earth; In the case of heating, the heat is extracted from the Earth and pumped into space.

A heat pump is governed by the same limitations of the second principle of thermodynamics (any energy transformation implies a dissipation of a heat-treated part that can no longer be used) like any other thermal and that maximum efficiency can be calculated from the Carnot cycle. Heat pumps are normally characterized by a performance coefficient that represents the ratio of its heating power to the electrical power absorbed by the grid.

High enthalpy geothermal energy is most commonly used to generate electricity. The typical geothermal system used to produce electricity has to produce about $10 \mathrm{~kg}$ of steam to produce a unit $(\mathrm{kWh})$ of electricity. The production of large amounts of electricity in the order of hundreds of megawatts requires the production of large volumes of fluid. Thus, one aspect of geothermal systems is that it must contain large amounts of high temperature fluid or a tank that can be recharged with fluids that are heated as contact with the rocks.

The three basic types of power generating installations are "dry" and "flash" central stations, where the hot water pressure (usually over) is low. The production of electricity in each type of installation depends on the temperatures and pressures of the tank and each type produces different environmental impacts. 


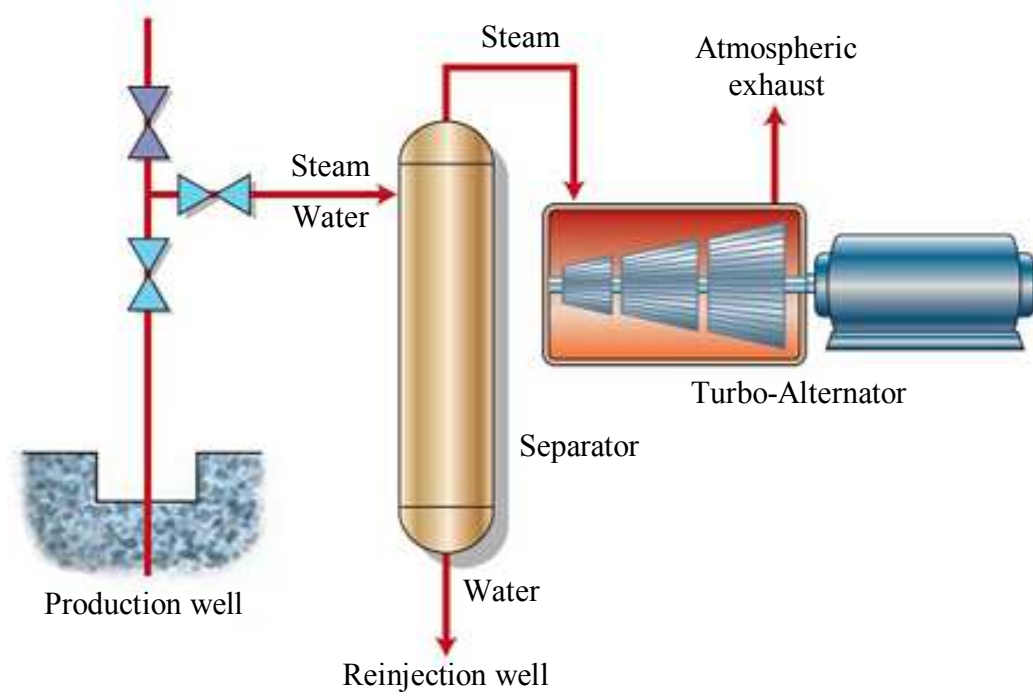

Fig. 6: Diagram of a back-pressure plant for electricity generation. The flow of the geothermal fluid is indicated in red. Source: (DiPippo, 2004)

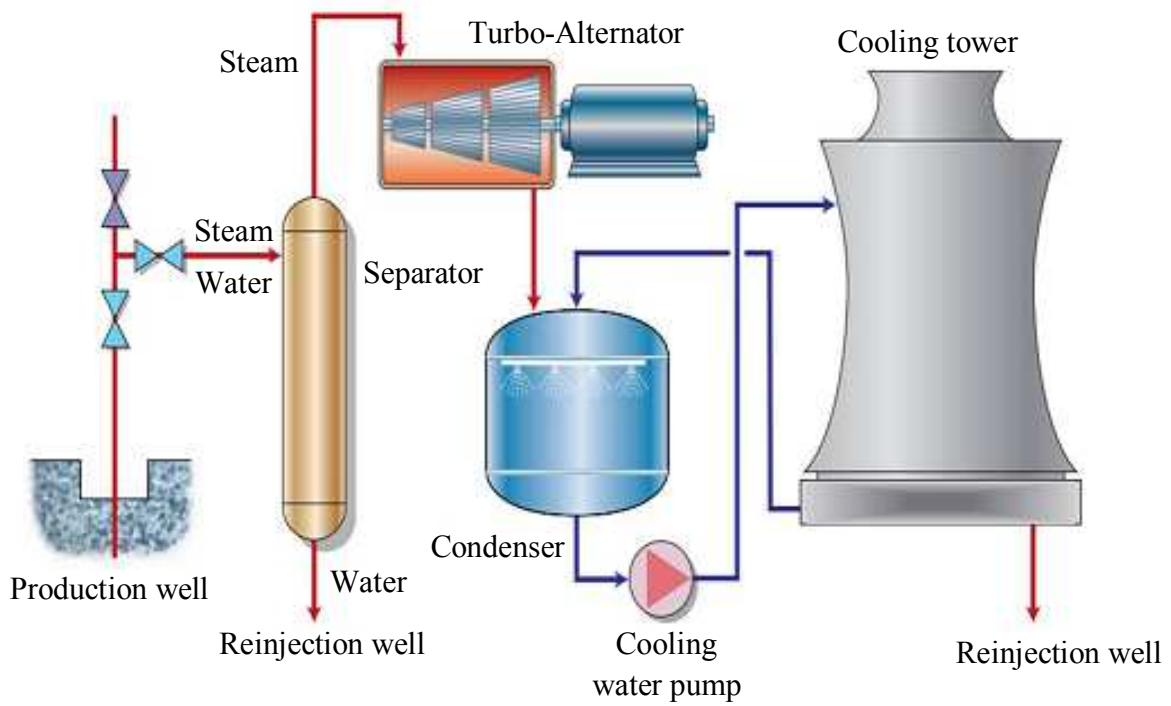

Fig. 7: Diagram of a condensation plan for the generation of electricity. The geothermal fluid circuit is indicated in red; the cooling circuit in blue. Source: (DiPippo, 2004)

The most common type of power plant today is the "flash" with water cooling system where a mixture of water and steam is produced by the spring. The steam is separated into a surface vessel and led to the turbine and the turbine trains a generator.

In a dry installation, the steam comes directly from the geothermal tank to the turbine that drives the generator and no separation is required because the source produces only steam.

In Fig. 9 one can see an example of a house heated by a typical ground-coupled heat pump system (Sanner et al., 2003).

Recent advances in geothermal technologies have made it possible to produce electricity in economically advantageous conditions and geothermal low temperature resources of $100-150^{\circ} \mathrm{C}$. Known as "binary" geothermal plants, these plants reduce emissions due to geothermal energy almost to zero. In the binary process, geothermal water heats another liquid, such as isobutane (most often n-pentane), which boils at a lower temperature than water and has high vapor pressure at low temperatures compared to steam. The two liquids are kept completely separated by using a heat exchanger to effect the transfer of thermal energy from geothermal water to the working fluid. The second fluid passes, vaporizes and turns into gaseous vapors and the force of expanding vapors drives the turbines that train the generators. 


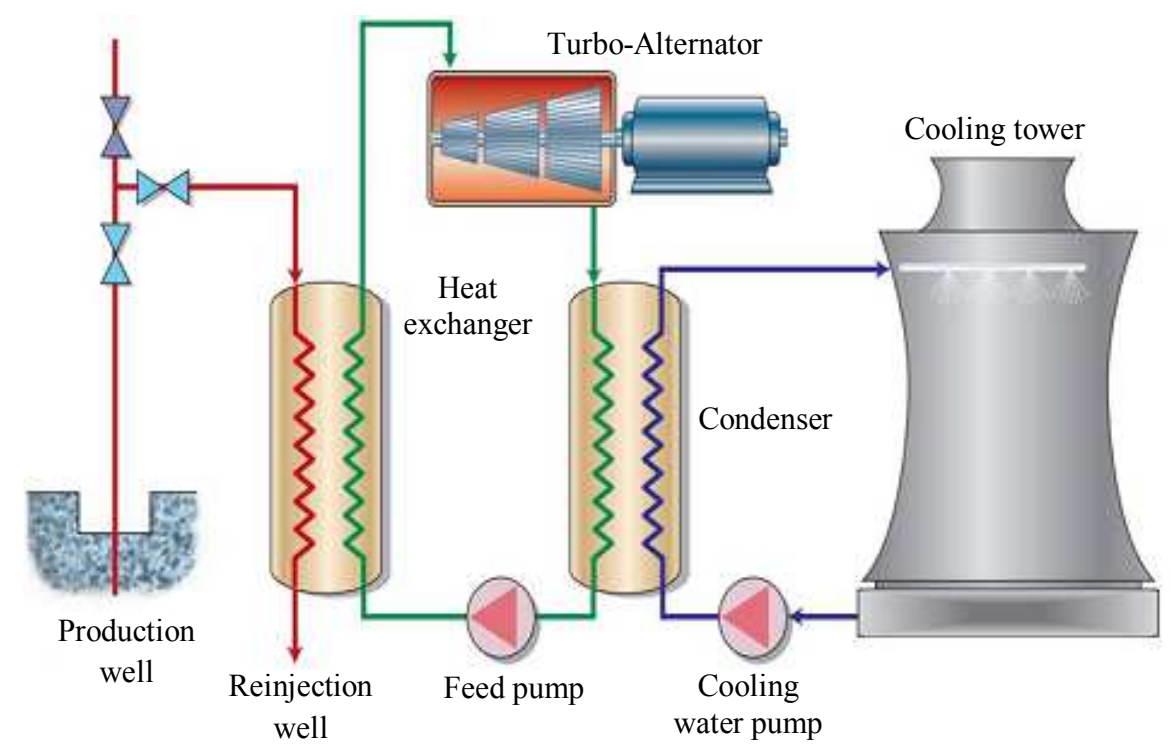

Fig. 8: Diagram of a binary cycle plant for electricity generation. The flow of the geothermal fluid is indicated in red; the secondary working fluid, in green; and the cooling circuit, in blue. Source: (DiPippo, 2004)

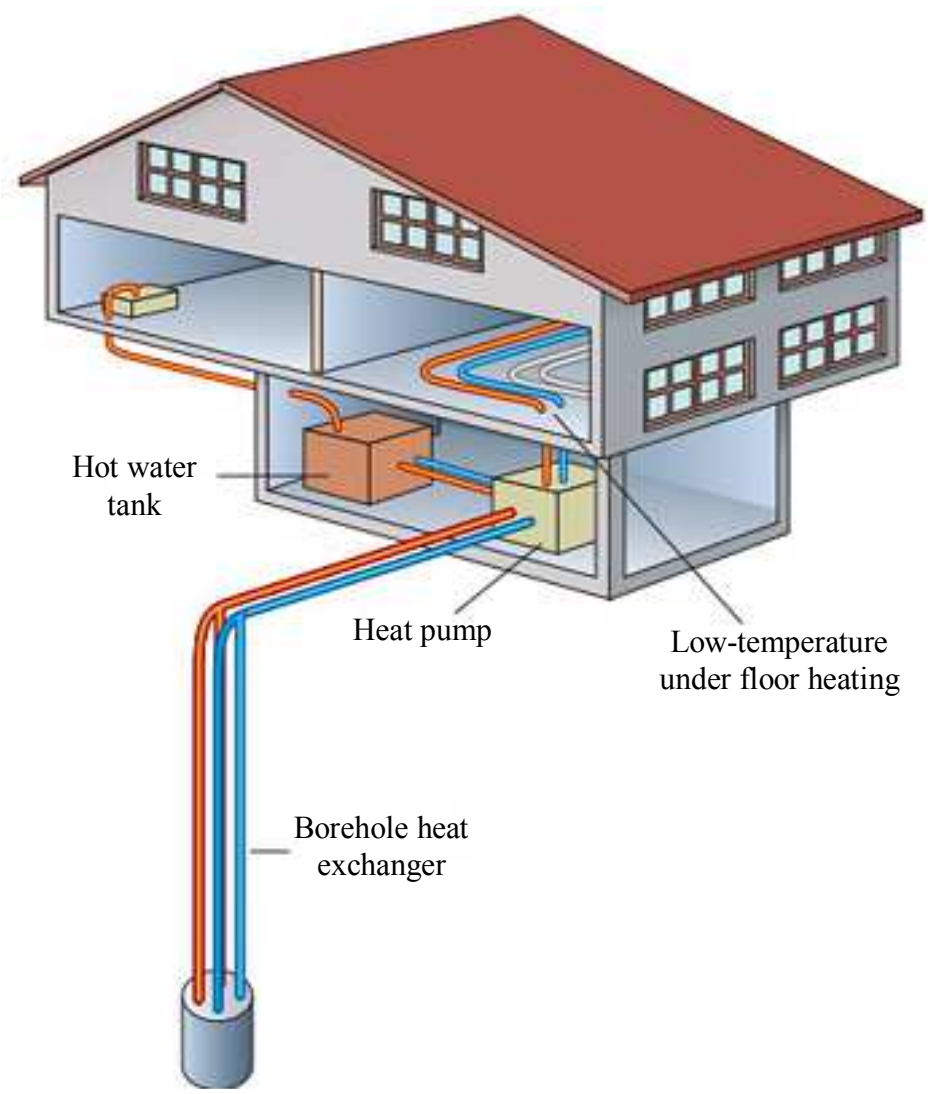

Fig. 9: Example of a house heated by a typical ground-coupled heat pump system. Source: (Sanner et al., 2003)

If the geothermal plant uses air cooling, geothermal fluids never make contact with the atmosphere before being pumped back into the underground geothermal reservoir. Developed in the 1980s, this technology is already in geothermal power stations in the world. The ability to use low-temperature resources increases the number of geothermal tanks that can be used to produce energy. 
Binary geothermal power stations, along with flash systems, produce almost zero emissions. In the case of direct use of thermal energy from geothermal hot water, the impact on the environment is negligible and can be easily reduced by adopting closed cycle systems, with the final extraction and re-injection of the fluid in the same geothermal reservoir.

The economic aspect of the use of hot water is still limiting for a wider spread in the energy sector. In fact, the economic benefit derives from its prolonged use over many years with low operating costs, although the initial investment may be considerable.

Identifying a geothermal reservoir is a complex activity consisting of different phases, starting from exploring the surface of a given area. This consists of the preliminary assessment of current geothermal events (hot water springs, steam jets, geysers, etc.), followed by geological, geochemical, geophysical and drilling exploration wells (several hundred meters deep) Measure the temperature (geothermal gradient) and evaluate the earth's heat flux. The interpretation of the collected data will suggest the location where deepwater exploration is to be carried out by well drilling (even at depths above $4000 \mathrm{~m}$ ) that will confirm the existence of geothermal fluids. In the case of positive results, the geothermal field that has been identified will be exploited by drilling a sufficient number of wells to produce geothermal fluid (hot water or steam).

The largest geothermal power plant in the world is in California - "Geysers", with an installed power of $750 \mathrm{MW}$.

Geological and hydrogeological studies are the starting point for any exploration program, the main purpose of which is to identify the location and size of the areas worth exploring in more detail and to recommend the most appropriate methods for exploring these areas. Geological and hydrogeological studies have an important role in all phases of geothermal research. They provide basic information for interpreting the data obtained with other exploration methods, and ultimately for the realization of a realistic model of the geothermal system and the assessment of the potential of the resource.

Geothermal areas should be further analyzed using geophysical techniques (gravimetry, magnetic and electrical tests, hot water chemical analysis, etc.) to locate specific reservoirs, the source of geothermal fluid. Geophysical analysis aims at indirectly determining physical parameters of depth geological formations from the surface or at depths close to the surface.

These physical parameters include:

- Temperature (temperature measurement)

- Electrical conductivity (electrical and electromagnetic methods)

- The propagation velocity of elastic waves (seismic studies)

- Density (gravity analysis)
- Magnetic susceptibility (magnetic methods)

Geothermal exploration is done through a sequence of several steps:

- Study on thermal conditions by collecting heat flow and map information

- Study on hydro-geological maps for assessing the distribution of groundwater resources

- Drilling wells for fluid extraction

Only after the surface exploration has shown that there is a workable potential, one can proceed to drill wells.

\section{Biomass}

Biomass is the "biodegradable fraction of products, wastes and residues of biological origin in agriculture (including vegetal and animal substances), forestry and related industries, including fisheries and aquaculture and the biodegradable fraction of industrial and municipal waste".

The benefits of biofuels compared to traditional fuels are aimed at greater energy security, lower environmental impact, currency savings and socioeconomic issues related to the rural sector. The concept of sustainable development embodies the idea of interconnectivity and balance between economic, social and environmental concerns.

The bioenergy production chain in a given territory must be achieved taking into account the technologies and types of biomass needed to achieve the best results. Therefore, the classification and characteristics of different biomass resources must be known.

An overwhelming part of biomass available for bioenergy comes from plant material and animal products. Some of the important features of different types of biomass are shown below. A first distinction can be made taking into account the origin of biomass from different sectors, such as agriculture, forestry, industrial and urban sectors. Another classification can be made by its nature: Energy crops, agricultural or forest residues and waste.

Biomass is mostly represented by energy crops from the agricultural and forestry sectors.

Herbaceous plants (monocots) represent the largest part of modern large-scale farming. Multi-annual grass crops include cereals such as grains, barley, oats, rye, other minor cereals: sugar beet, sugarcane, fodder crops and clover. The seeds of these cereals, stems and tubers of other plants are a good source of starch that can be used in technological processes for energy production and biofuels.

Selective reproduction (especially for non-food crops) has been used to modify seed/plant ratio for many biomass species with high seed production.

This type of biomass can be used as a raw material for the production of bioenergy when economically 
viable. Rapid reed and cane species are examples of grassy crops that can make good use of available nutrients to increase biomass productivity; But at the same time other agronomic features still present weaknesses, such as floral sterility, prohibitive costs for setting up culture, relatively low harvest mechanization, high humidity of the harvested product and high ash content.

Oil crops include annual crops of oilseeds and oleaginous perennial crops. The most representative oilseed crop in the European regions is sunflower and soybean. Vegetable oil is typically extracted by mechanical pressing and/or solvent extraction and is used in the food industry, soap and cosmetics. Oils in these crops also contain other seed constituents (proteins or starch). The lignocellulosic part of oleaginous crops, which is traditionally used as a mulch or feed, can also be burned to produce energy or heat, while vegetable oils can be used for higher value bioenergy applications, especially as a substitute for Diesel fuel.

Vegetable oils derived from these cultures and modified in m-methyl esters are commonly referred to as "biodiesel" and are the main candidates for becoming alternative fuels. But the use of edible oils for energy purposes can cause significant problems, such as hunger in developing countries. The double use of palm oil increases competition between the edible oil and biofuel market, resulting in higher prices of vegetable oil in developing countries.

The use of non-edible vegetable oils when compared to edible oils is very significant in developing countries because of the huge demand for edible oils, which are far too expensive to use as fuel today. The production of biodiesel from various non-edible oils has been intensively researched in recent years.

Residue and waste biomass analysis is more complicated due to the variety of materials and sectors of origin (from agriculture to urban).

Wastes are those generated in the production process, industrial waste and solid municipal waste. The typical energy content is from 10.5 to $11.5 \mathrm{MJ} / \mathrm{kg}$. Waste management practices vary from country to country, from urban areas to rural areas, from industrial to residential.

The situation of waste management in a developing country differs from that of an industrialized country. Transfer of technology from one country to another may be totally inappropriate, although technically the technology is viable and accessible.

It is very important to understand local factors such as:

- Characteristics and seasonal variations of waste

- Social issues related to habits regarding solid waste and the attitude of political institutions

- Awareness of other resource limitations the role of sustainable waste management is to reduce the amount of waste released into the environment by reducing the amount of waste produced
Large quantities of waste can't be eliminated. However, the impact on the environment can be reduced by sustainable waste use. This is known as the "waste management hierarchy". The waste management hierarchy refers to reduction, re-use and recycling and the classification of waste management strategies according to their desirability to minimize waste. The purpose of the waste management hierarchy is to obtain the maximum practical benefits of a product and to generate a minimal amount of waste.

Biomass from residues and wastes includes plant and animal waste. These are agricultural residues such as straw, vegetable and fruit shells, residues and forest waste, such as leaf layer, sawdust residues, food waste and the organic solid waste mining component. Energy can be generated from these wastes, as globally there are several billion tons of biomass contained in them.

There are many options available for converting waste and waste into energy. These technologies are: Waste disposal, incineration, pyrolysis, gasification, anerobic digestion and others. Energy density and physical properties of biomass are critical factors for the raw material and need to be understood in order to choose the processing technology.

The choice of technology must be based on the type of waste, its quality and local conditions, but a classification of different types of waste is not easy. In the countries of the European Union waste is classified according to the European Waste Catalog.

In recent years, the production of energy and biofuels from waste and residues has become very important due to the positive economic and environmental effect. The use of urban waste for energy purposes could avoid an increase in the surface of urban waste landfills, resulting in a reduction in greenhouse gas emissions and greater independence from fossil fuels.

Major agricultural waste includes plant residues, straw and shells, olive stones and nut shells. More specifically, residues can be divided into two general categories: - Waste from the field: The material remaining in the field or in orchards after harvestings, such as cocci, stems, leaves and seed pods. - Processing residues: Material left after harvest processing, shells, seeds, roots.

Much of the wood from the forest sector is a major source in some countries and is used as the main fuel for small-scale energy production in rural areas, where gas heating is unusual. Wood is thus a competitor for fossil fuels and is used both in the household for cooking and heating water, as well as in industrial and commercial processes (for water heating or process thermal energy). The alternative to the use of waste from the forestry sector or related industrial activities, such as timber factories, is an attractive source of biomass and a successful example for the generation of waste energy. Forest residues are wood from cuttings, logging residues, trees, shrubs, tree bark, etc. Normally forest residues are 
considered to be a better fuel than agricultural residues, but their density and the collection system (especially when the land is high) lead to a high cost of transport; The net $\mathrm{CO} 2$ emission produced for each unit of energy provided by forest residues is lower than that produced by other agricultural waste due to fertilizers and pesticides used in agriculture.

The energy content of different vegetable materials determines their calorific value. The calorific value depends on the percentage of carbon and hydrogen, which are the main contributors to the biomass energy value.

In order to obtain maximum energy, plant materials should be dried, as the amount of energy contained in plants varies depending on the moisture content. In the case of firewood, the calorific value decreases linearly with the increase in moisture content.

The biomass potential is the total amount of source that is present on a given territory; It is common to refer to the potential of biomass in several ways: Theoretically, technically, ecologically and economically. In practical terms, biomass actually available for energy uses derives from the application of certain restrictions (technical, environmental, other restrictions on land use competition) to the theoretical potential.

Boiler combustion is the most widespread biomass energy utilization technology. Types of boilers for combustion of wood biomass are very varied and could be classified into three groups:

(1) Boilers with grilling

(2) Boilers with under floor heating

Bias gasification is a complete gas conversion process using air, oxygen or steam gasification media. The biomass gasification is achieved by two main methods:

- Thermal gasification using air, oxygen, steam or their mixture at temperatures of about $7000 \mathrm{C}$

- Biochemical gasification using micro-organisms at ambient temperature and under anaerobic conditions

For the gasification of wood, three main types of gasification reactors were developed and applied:

- $\quad$ Fixed bed gasogens

- $\quad$ Fluidized bed gasifiers

- Ascending current gas

\section{Energy from Water}

From the water, we can extract energy in many ways than the already known classics.

I-A special symbiosis is that between hydroelectric power plants (using storage systems) and wind energy.
When the wind energy produced is large, it can be used to store energy by upstream water lifting by means of electric pumps powered by surplus electricity produced by one or more windmills. When the energy consumption is high, the water stored at the height is left to fall to produce an energy surplus that will be directly distributed to the respective national energy system (Petrescu et al., 2016a).

II-Nuclear fusion, sometimes referred to as thermonuclear fusion, is a process where two light atomic nuclei come together to form a heavier nucleus. This reaction is at work in a natural way in the Sun and most stars of the Universe.

The fusion of light nuclei releases enormous amounts of energy from the attraction between the nucleons due to the strong interaction (nuclear binding energy). Fusion it is with nuclear fission one of the two main types of nuclear reactions applied (Shultis and Faw, 2002).

The mass of the new atom obtained by the fusion is less than the sum of the masses of the two light atoms. In the process of fusion, part of the mass is transformed into energy in its simplest form: heat. This loss is explained by the Einstein known formula $\mathrm{E}=\mathrm{mc}^{2}$.

One of its interests is to be able to obtain theoretically much more energy: first to mass of "fuel" equal, the fusion releases three to four times more energy than fission. Then, the "fuel" stock is much larger: the oceans naturally contain such a mass of deuterium (33 $\mathrm{g} / \mathrm{m}^{3}$ ) that they could theoretically satisfy the current energy consumption of the human species for one hundred million Years $\left(1 \mathrm{~m}^{3}\right.$ of water can potentially provide as much energy as the combustion of $700 \mathrm{t}$ of oil).

Despite research carried out around the world since the 1950s, no industrial application of fusion to energy production has yet succeeded, apart from nuclear weapons with the H-bomb, since this application does not Aims at containing and controlling the reaction produced. There are, however, some other less mediated uses, such as neutron generators (Thermonuclear weapon, from Wikipedia).

Unlike nuclear fission, the fusion products themselves (mainly helium 4) are not radioactive, but when the reaction is used to emit fast neutrons, they can transform the nuclei that capture them into isotopes that some of them can be radioactive.

We must not confuse nuclear fusion with the fusion of the core of a nuclear reactor, which is a particularly formidable nuclear accident.

The only nuclear reaction carried out on an industrial scale up to now is that of nuclear fission. With all that there were some nuclear accidents and the raw material used as fuel and residue also are radioactive, energy from nuclear fission represented for humanity a necessary evil. She managed to prevent an energy major crisis for humanity.

We were not allowed back in the caves as it has started with millions of years ago. We do not want to give up nor to our current homes comfortable, that we 
make them to be warm in winter and cooling them in summer you (to feel us better).

The energy for fission helped us to avoid a crisis disaster and in addition to prolong the life of the hydrocarbons. Today we are a little better.

The advanced technologies allowed us to implement new renewable energies, sustainable, green and friendly for man and to the environment. It is better to continue to implement other and other central solar energy, wind or hydro. But if we have a moment of rest, it does not mean that we must give up to a beautiful dream of humanity, namely the one to bring the sun on the Earth. In other words, we must continue to try to devise and achieve the nuclear fusion, industrial and peaceful (Halliday and Robert, 1966). A let us begin by presenting a wellknown diagram among specialists (Fig. 10).

Stable nuclei are represented by filled circles (blackened). Radioactive nuclei (i.e., those which spontaneously decay, emitting electrons or alpha particles) are represented by unfilled circles.

On the $\mathrm{x}$-axis was represented atomic number $Z$ (indicate number of protons in the nucleus).

On the $y$-axis was represented the number of the neutronic $N$ (which indicate number of neutrons in the nucleus; Progress in Fusion, ITER).

It is important to note that almost every known element from the Mendeleev's table of chemical exists in several forms, in reality, called isotopes. To understand better what are isotopes of an element, consider for example iron (to follow the arrow that indicates iron isotopes), which has 26 permanent protons $(Z=26)$, but it may have in its various isotopes (eight in number) various structures neutron $(N=26-33)$. The iron has four isotopes stable and four radioactive.

The next logical step is to try to determine the approximate Coulomb force acting between two protons in the atom of iron.

Using heavy water, it is hoped that future infinite energy can be extracted by producing a controlled fusion reaction (Petrescu et al., 2016b).

It is known that not only the second isotope of hydrogen (Deuterium) can produce fusion nuclear energy, but and the third (heavy) isotope of hydrogen (Tritium) may produce energy through a nuclear fusion.

First nuclear fusion reaction it is possible between two nuclei of Deuterium and may be obtained: One Tritium nucleus plus a proton and energy, either a helium isotope with a neutron and energy (Equation 1 and 2), (Petrescu, 2012; Petrescu and Calautit, 2016a; Petrescu and Petrescu, 2012; Petrescu et al., 2016):

$$
\begin{aligned}
& { }_{1}^{2} \mathrm{D}+{ }_{1}^{2} \mathrm{D} \rightarrow{ }_{1}^{3} \mathrm{~T}+1.01 \mathrm{MeV}+{ }_{1}^{1} \mathrm{H}+3.02 \mathrm{MeV} \\
& ={ }_{1}^{3} \mathrm{~T}+{ }_{1}^{1} \mathrm{H}+4.03 \mathrm{MeV} \\
& { }_{1}^{2} \mathrm{D}+{ }_{1}^{2} \mathrm{D} \rightarrow{ }_{2}^{3} \mathrm{He}+0.82 \mathrm{MeV}+{ }^{1} n+2.45 \mathrm{MeV} \\
& ={ }_{2}^{3} \mathrm{He}+{ }^{1} n+3.27 \mathrm{MeV}
\end{aligned}
$$

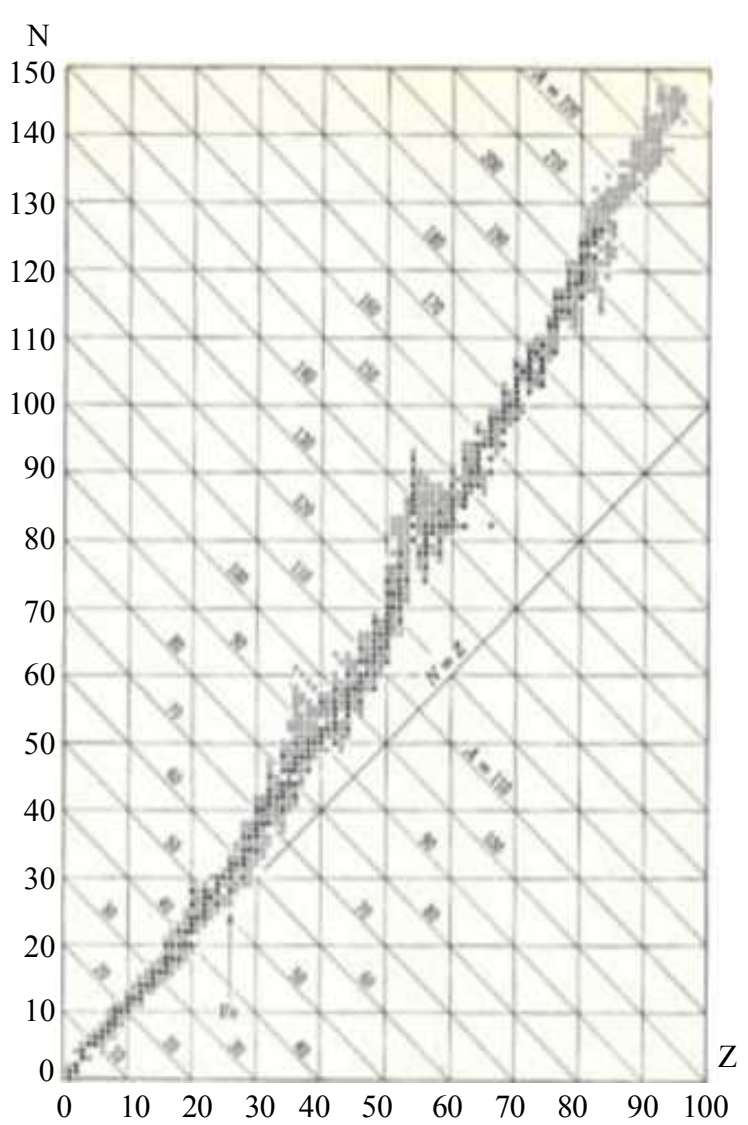

Fig. 10: Diagram of atomic cores (atomic nuclei); Source: (Halliday and Robert, 1966)

Fusion reaction may occur and between a nucleus of Deuterium and one of Tritium (Equation 3) and this fusion nuclear reaction may be produced more easily than one between two deuterons (Equation 1 and 2) (Krane, 1987):

${ }_{1}^{2} \mathrm{D}+{ }_{1}^{3} \mathrm{~T} \rightarrow{ }_{2}^{4} \mathrm{He}+3.5 \mathrm{MeV}+{ }^{1} n+14.1 \mathrm{MeV}$
$={ }_{2}^{4} \mathrm{He}+{ }^{1} n+17.6 \mathrm{MeV}$

An important nuclear reaction may be produced between a nucleus of Deuterium and an isotope of Helium (Equation 4):

$$
\begin{aligned}
& { }_{1}^{2} \mathrm{D}+{ }_{2}^{3} \mathrm{He} \rightarrow{ }_{2}^{4} \mathrm{He}+3.6 \mathrm{MeV}+{ }_{1}^{1} \mathrm{H}+14.7 \mathrm{MeV} \\
& ={ }_{2}^{4} \mathrm{He}+{ }_{1}^{1} \mathrm{H}+18.3 \mathrm{MeV}
\end{aligned}
$$

The isotope of helium ${ }_{2}^{3} \mathrm{He}$ is obtained in the reaction of 2 .

The reaction of the 1 generates Tritium which together with Deuterium (if one of them or both have enough energy) produce the nuclear reaction of 3 to generate a lot of energy and helium, a non toxic, inert and very stable gas. 
For this reason the group of nuclear reactions of fusion is an advantageous one, friendly, pure, peacefully and inexpensive.

Naturally the Tritium appears in the nuclear reactor, only when the reaction of 1 is produced, but one may obtains more Tritium from the nuclear reaction (Equation 5):

$$
{ }_{3}^{6} \mathrm{Li}+{ }^{1} n \rightarrow{ }_{1}^{3} \mathrm{~T}+{ }_{2}^{4} \mathrm{He}+4.784 \mathrm{MeV}
$$

The bars of lithium are easily entered or extracted in the nuclear reactor and by this mechanism can be controlled very simple and the fusion reaction speed at any time.

The reaction of the 5 can help much the reaction to the merger, by controlling its production. Lithium reserves in the earth's crust would permit the operation of melting plants for more than 1,000 years and those of the oceans could meet the needs for millions of years.

Neutrons necessary for producing the reaction 5 are generated even in the reactor in the framework of the reactions 2 and 3 (Petrescu, 2012; Petrescu and Calautit, 2016a; Petrescu and Petrescu, 2012; Petrescu et al., 2016).

Raw materials to achieve nuclear fusion are the deuterium and lithium, more exactly heavy water and bars of lithium. Result a lot of energy and helium.

The reaction can be controlled easily through various methods. The reaction of the merger no tends to "get out of control" such as to the fission (being difficult to start it and easy to stop it), (Shultis and Faw, 2002).

Equation 6 can generates extra energy and it is much easier to achieve on the Earth than other possibilities:

$$
{ }_{1}^{3} T+{ }_{1}^{3} \mathrm{~T} \rightarrow{ }_{2}^{4} \mathrm{He}+2 \cdot{ }^{1} n+11.3 \mathrm{MeV}
$$

In a nuclear reactor of this type, supplied with Deuterium, can take place and other nuclear reactions, of which the most important are (Equation 7-9):

$$
\begin{aligned}
& { }_{2}^{3} \mathrm{He}+{ }_{2}^{3} \mathrm{He} \rightarrow{ }_{2}^{4} \mathrm{He}+2 \cdot{ }_{1}^{1} \mathrm{H}+12.9 \mathrm{MeV} \\
& { }_{2}^{3} \mathrm{He}+{ }_{1}^{3} \mathrm{~T} \rightarrow{ }_{2}^{4} \mathrm{He}+{ }_{1}^{1} \mathrm{H}+{ }^{1} \mathrm{n}+12.1 \mathrm{MeV} \\
& { }_{2}^{3} \mathrm{He}+{ }_{1}^{3} \mathrm{~T} \rightarrow{ }_{2}^{4} \mathrm{He}+4.8 \mathrm{MeV}+{ }_{1}^{2} \mathrm{D}+9.5 \mathrm{MeV} \\
& ={ }_{2}^{4} \mathrm{He}+{ }_{1}^{2} \mathrm{D}+14.3 \mathrm{MeV}
\end{aligned}
$$

Lithium with deuterium can still generate four other important reactions (Equation 10-13):

$$
\begin{aligned}
& { }_{1}^{2} \mathrm{D}+{ }_{3}^{6} \mathrm{Li} \rightarrow 2 \cdot{ }_{2}^{4} \mathrm{He}+22.4 \mathrm{MeV} \\
& { }_{1}^{2} \mathrm{D}+{ }_{3}^{6} \mathrm{Li} \rightarrow{ }_{2}^{3} \mathrm{He}+{ }_{2}^{4} \mathrm{He}+{ }^{1} n+2.56 \mathrm{MeV} \\
& { }_{1}^{2} \mathrm{D}+{ }_{3}^{6} \mathrm{Li} \rightarrow{ }_{3}^{7} \mathrm{Li}+{ }_{1}^{1} \mathrm{H}+5.0 \mathrm{MeV}
\end{aligned}
$$

${ }_{1}^{2} \mathrm{D}+{ }_{3}^{6} \mathrm{Li} \rightarrow{ }_{4}^{7} \mathrm{Be}+{ }^{1} \mathrm{n}+3.4 \mathrm{MeV}$

Lithium can react and with hydrogen $=$ Protium $(\mathrm{a}$ proton; Equation 14) or with an isotope of $\mathrm{He}$ (Equation 15):

$$
\begin{aligned}
& { }_{1}^{1} \mathrm{H}+{ }_{3}^{6} \mathrm{Li} \rightarrow{ }_{2}^{4} \mathrm{He}+1.7 \mathrm{MeV}+{ }_{2}^{3} \mathrm{He}+2.3 \mathrm{MeV} \\
& ={ }_{2}^{4} \mathrm{He}+{ }_{2}^{3} \mathrm{He}+4.0 \mathrm{MeV} \\
& { }_{2}^{3} \mathrm{He}+{ }_{3}^{6} \mathrm{Li} \rightarrow 2 \cdot{ }_{2}^{4} \mathrm{He}+{ }_{1}^{1} \mathrm{H}+16.9 \mathrm{MeV}
\end{aligned}
$$

It should also be mentioned separately an extremely exciting nuclear reaction (Equation 16) that may occur between the stable isotope of boron with 6 neutrons (boron has five protons) and the first isotope of hydrogen, Protium (Hydrogen, from Wikipedia).

The reaction between hydrogen and boron can be achieved more easily than others and can generate a large amount of energy plus the inert gas, $\mathrm{He}$ :

$$
{ }_{1}^{1} \mathrm{H}+{ }_{5}^{11} \mathrm{~B} \rightarrow 3 \cdot{ }_{2}^{4} \mathrm{He}+8.7 \mathrm{MeV}
$$

If the reaction of merger Protium-Protium can be produced only in the stars (as the Protium is a very stable isotope) on the Earth we can try the easiest to achieve the merger Tritium-Tritium (Equation 6), as the Tritium is an isotope unstable (see diagram of the Fig. 1).

In the laboratory may be carried out and the reactions between Protium and Boron (Equation 16), or Protium and Lithium (Equation 14).

Today we can hope to get infinite energy from the water through a simple, controlled process. It is about water split into hydrogen and oxygen by modern nano methods, using ultraviolet radiation, so that the energy used to separate water into its components being lower than that obtained then by burning hydrogen. Many experts work in this area and have already achieved remarkable results.

Once this process will have been put in place, it will allow water to be used in the future as an energy storage and storage agent.

Today we can extract wave and tidal energy.

\section{High-Powered Stations}

Tidal energy is the energy that can be captured by exploiting the potential energy resulting from the vertical displacement of the water mass at different levels or the kinetic energy due to the tidal currents.

Tidal energy is a consequence of solar and lunar gravitational forces, as well as a consequence of ground rotation.

Large-scale power plants are of two types:

- Central without a dam 
- Which uses only the kinetic energy of tidal current

- Central dam

- Which uses the potential energy created as a result of the water level rise during the flow

Power plants using wave energy.

Waves are formed due to the action of moving air masses (wind) on the water. The magnitude of the waves generated depends on the wind speed, its duration and distance on the water (length of action).

The water movement resulting from this action has a kinetic energy exploitable with the help of special technologies.

Wave energy capture systems are of three types:

- The pressure piping system that is based on the fact that a pressure exerted on a large surface and transmitted by a liquid through pipes to a smaller surface multiplies the force on the surface unit and this force is used to drive an electric generator

- The system based on liquid ascension

- Which uses the rise of water as a wave on an artificial slope, then the turbine of an electric generator

- The liquid piston system

- Which is based on the fact that in an enclosure the waves produce by their climbing and lowering movement a similar effect to a piston pumping or aspiring the air acting on the turbine of an electric generator

\section{Disadvantages}

Wave and tidal power plants can have a negative impact on marine ecosystems and coastal areas (Fig. 11).

\section{Osmotic Energy}

Potential osmotic energy or salinity gradient energy is the energy obtained from the salt concentration difference between sea water and river or river water. Two practical methods for this are reverse inverse electrode (EDI) and delayed pressure Osmosis (OIP).

Both processes are based on osmosis with specific ion membranes, the resulting residual product being salty water.

The osmotic energy utilization is based on the osmotic pressure difference between freshwater and seawater (Fig. 12).

The first osmotic power plant, with a installed power of $4 \mathrm{~kW}$, was built in Norway by Statkraft and became operational on 24 November 2009. In this plant the energy is obtained on the basis of a flow rate of $10 \mathrm{l} / \mathrm{s}$ flowing through membrane at a pressure of 10 bar.

Increasing water flow and increasing pressure will increase the capacity to generate electricity.

\section{Disadvantages}

Drainage resulting as a residual product may have adverse effects on the environment.

\section{Other Technologies that use Air Energy}

\section{Compressed Air Engine}

A compressed air engine is a pneumatic actuating mechanism that generates mechanical work by releasing the air in a pressure tank (Fig. 13).

There are several types of such engines, ranging from small engines to several hundred horsepower engines.

Some types are based on pistons and cylinders, others use turbines.

Later, several types of vehicles with compressed air powered engines have been built, but their disadvantage is low speed and reduced autonomy.

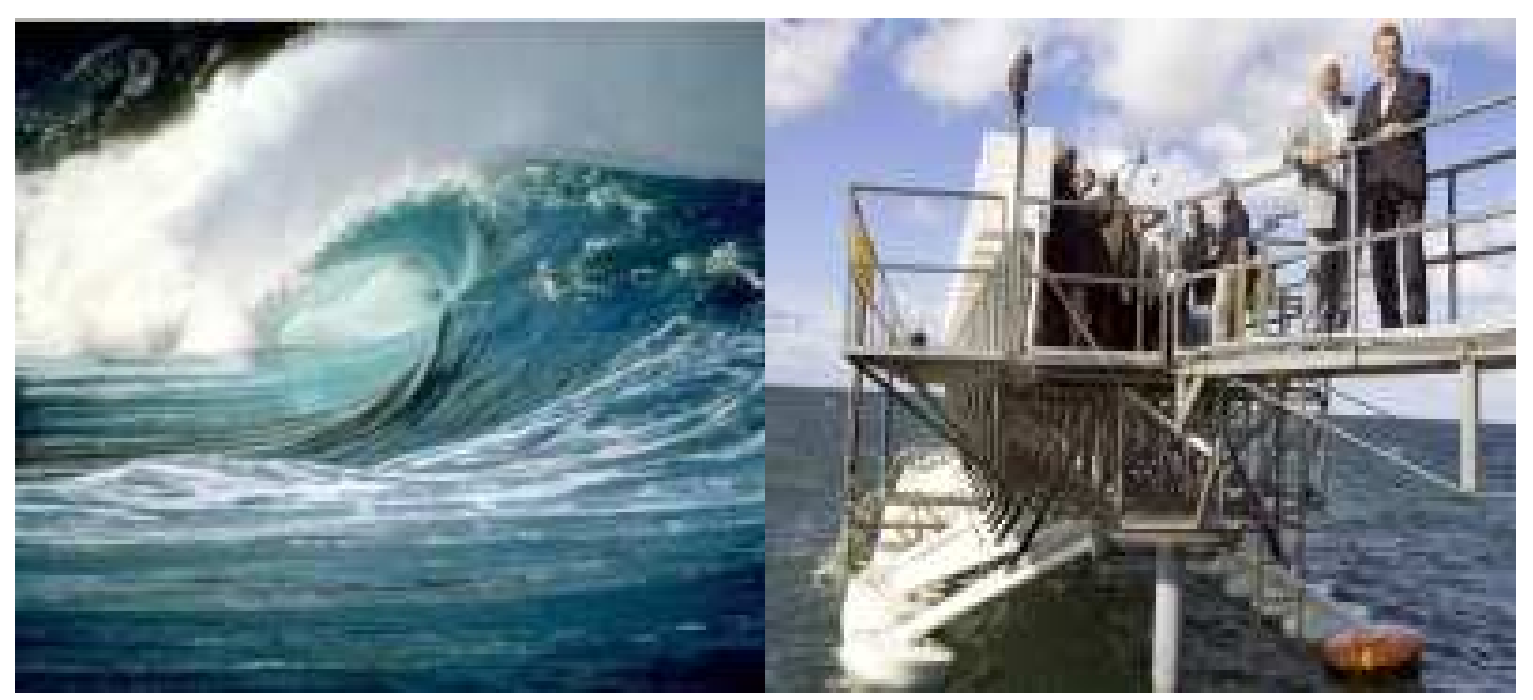


Relly Victoria Virgil Petrescu et al. / American Journal of Engineering and Applied Sciences 2017, 10 (4): 919.948

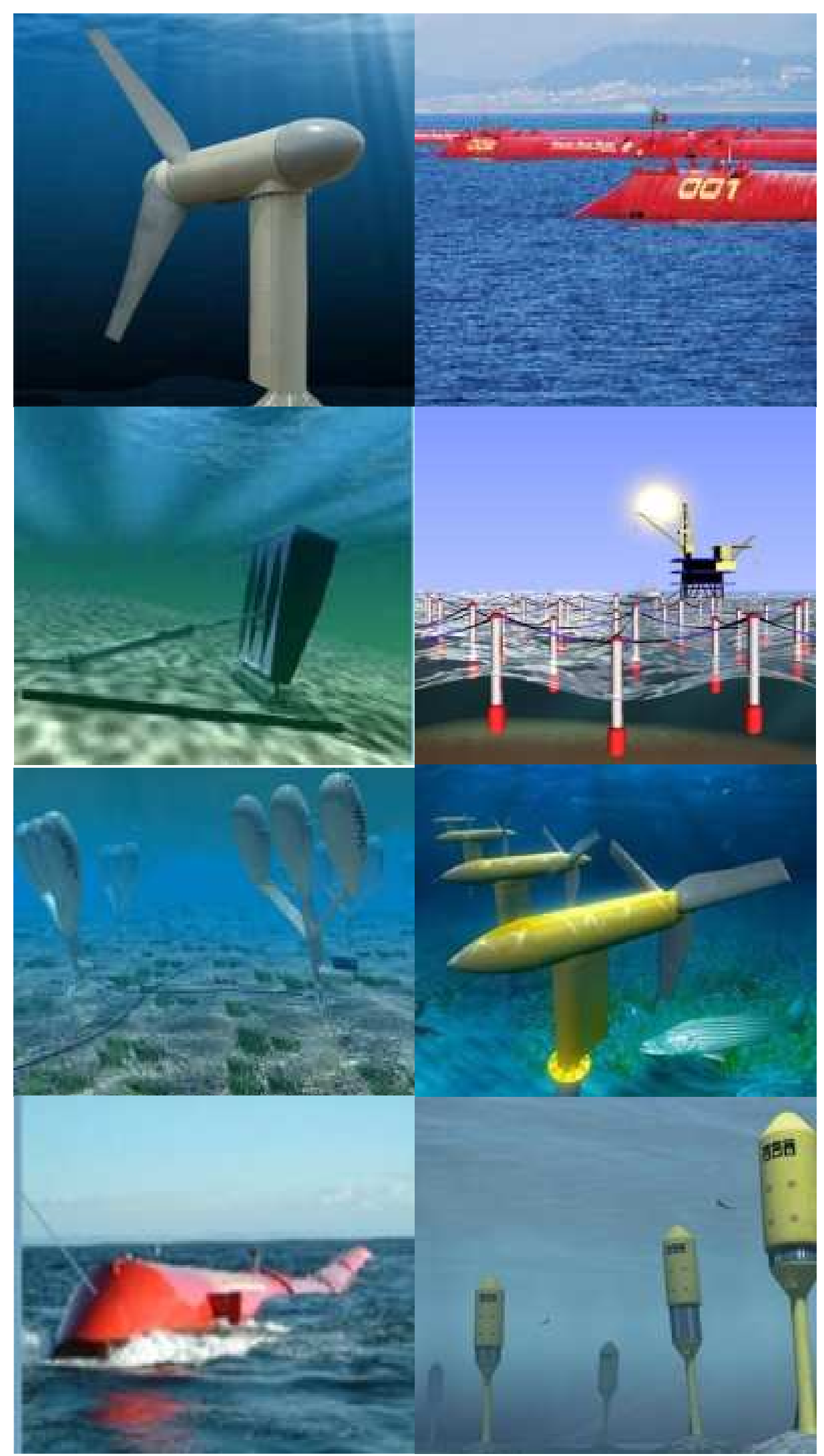

Fig. 11: Wave and tidal energy 


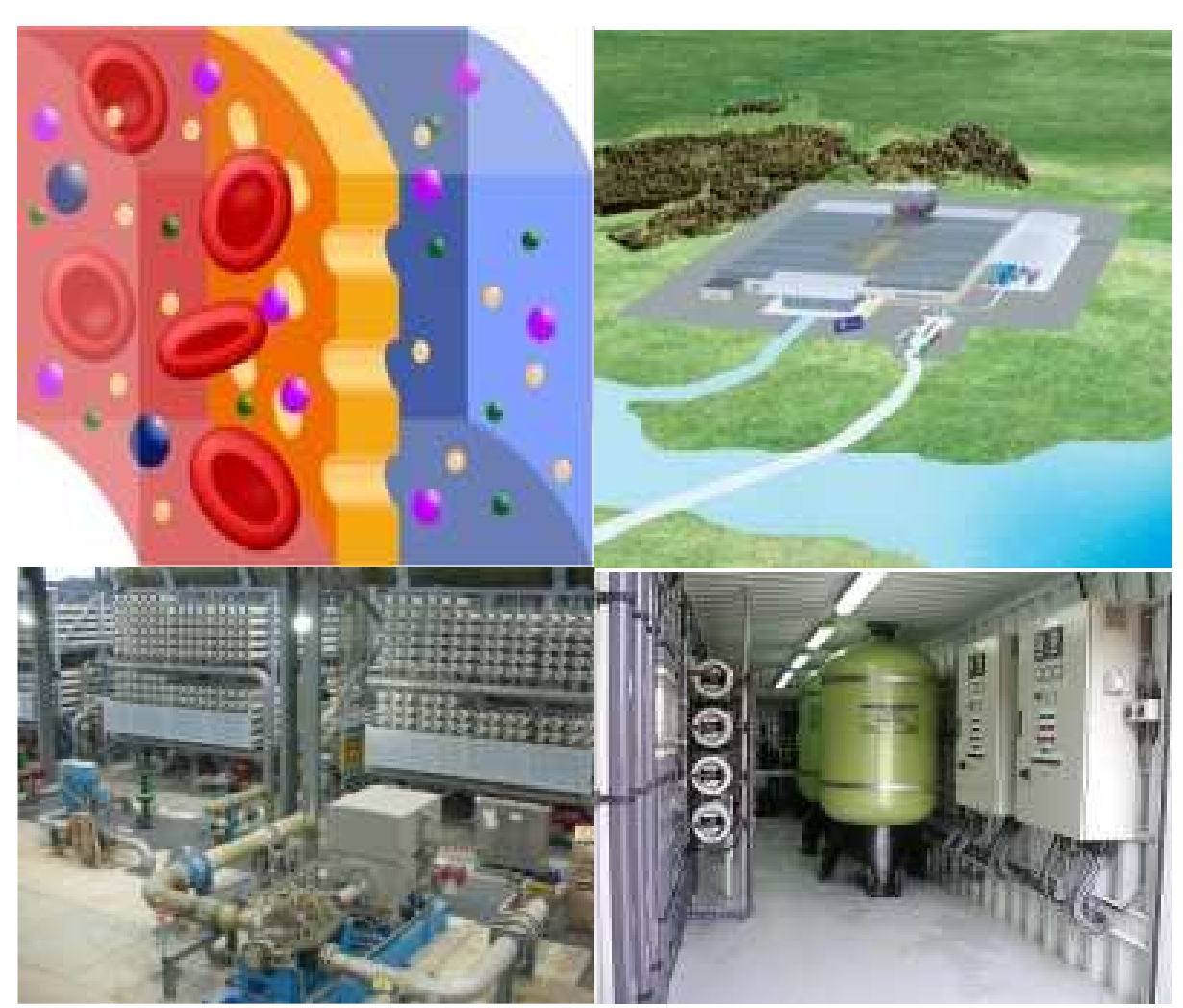

Fig. 12: Osmotic energy
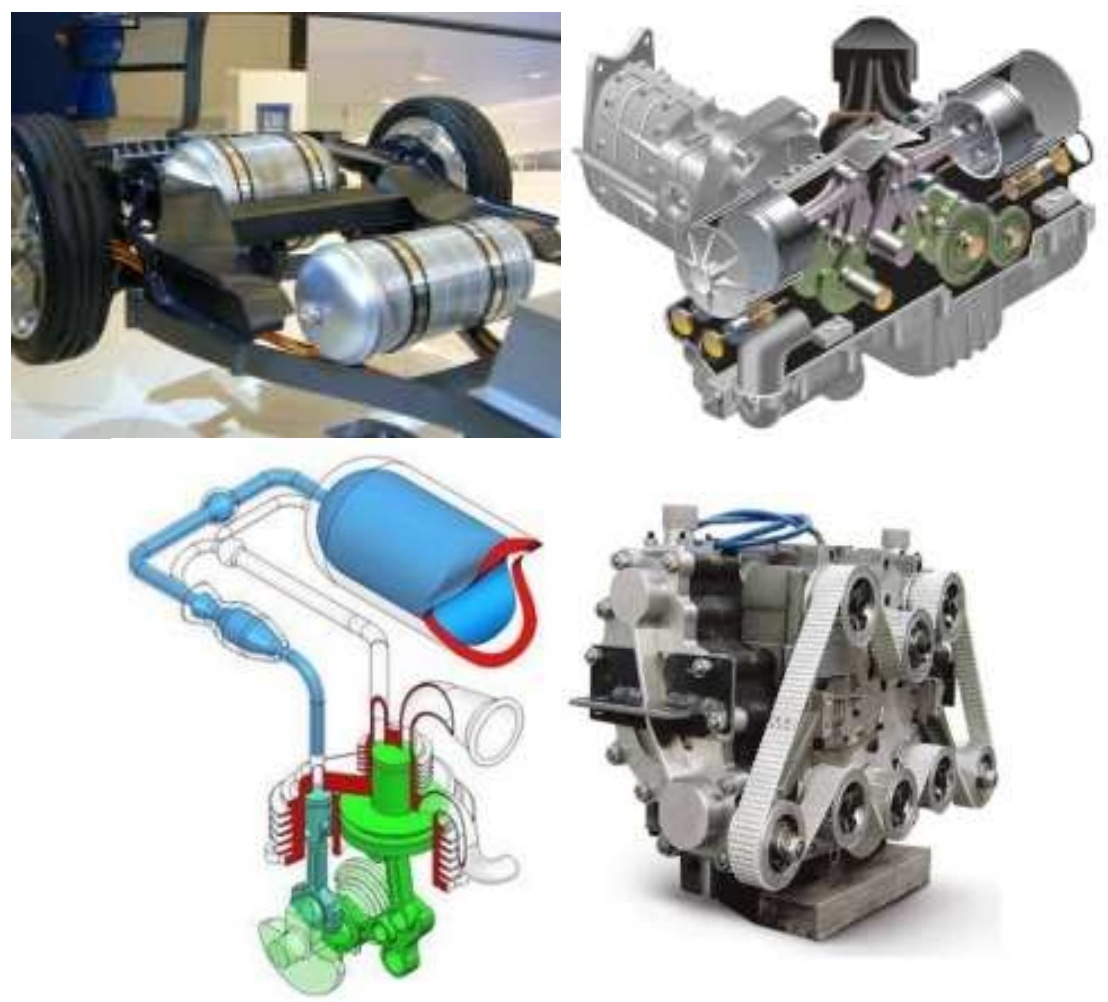

Fig. 13: A compressed air engine is a pneumatic actuating mechanism that generates mechanical work by releasing the air in a pressure tank 


\section{Magnet-Based Systems without Moving Parts}

Magnetic energy generators with no moving parts are based on permanent magnets coupled in different ways with ferromagnetic cores and whose magnetic field interacts with magnetic fields generated by electroniccontrolled coil groups (Fig. 14).

There are many such patented devices, systems and generators both scientists and amateurs over time. Among them, we mention:

- The electric generator built by Graham Gunderson
- Charles Flyn's device

- Lawrence Tseung's device

- Hans Coler's magneto generator

The electromagnetic Generator (MEG) built by Tom Bearden, Stephen Patrick, James Hayes, Kenneth Moore and James Kenny:

- Thane Heins' transformer

- Richard Willis's generator

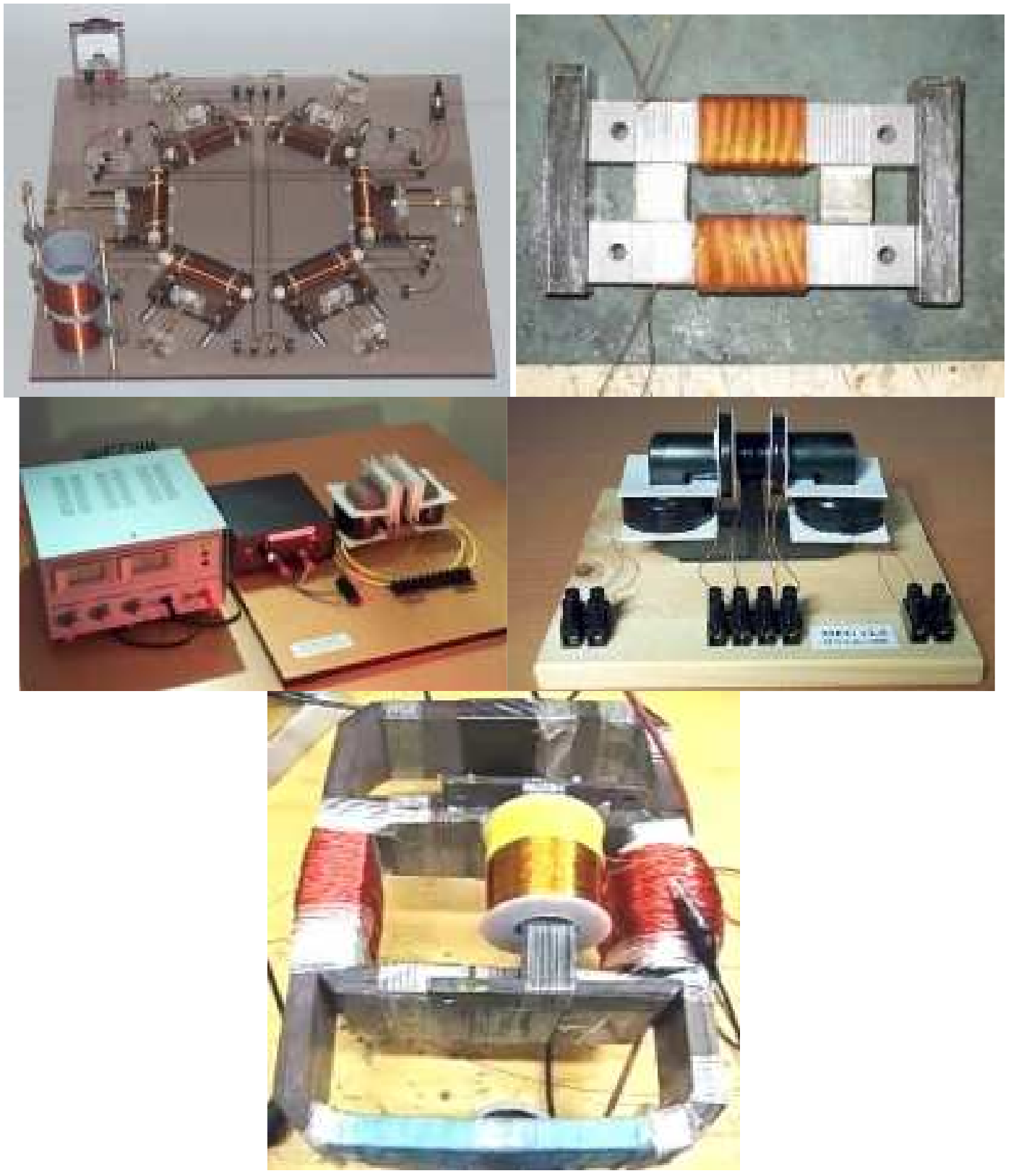

Fig. 14: Magnetic energy generators with no moving parts are based on permanent magnets coupled in different ways with ferromagnetic cores and whose magnetic field interacts with magnetic fields generated by electronic-controlled coil groups 


\section{Results}

To address an investment in RES, selecting locations favorable to energy applications is made taking into account some criteria, including technical, economic and environmental conditions and restrictions. The main selection criteria are as follows:

1) The energy potential of the renewable source in the area of interest

2) Concrete conditions in the field (soil morphology, roughness, obtacole, nature of the land)

3) Approaching human settlements

4) Nature reserves, historic, tourist, archaeological areas

5) Special landmarks: Forbidden areas, civil/military airport, special telecommunication targets, etc.

6) The existence and status of access paths

7) Terms of use of the land: Legal regime, concession/purchase

8) Electrical connection possibilities: Distance, power level, etc

9) The existence of a consumer in the area

10) Potential investors in the area

11) Potential auto producers in the area

12) The possibility of a public/private partnership

13) Technical and economic performance indicators favorable to the investment approach in the selected site

Wind energy is generated by the wind energy transfer of a wind turbine. The winds are formed by the uneven heating of the Earth's surface by the energy radiated by the Sun that reaches the surface of our planet. This variable heating of the air layers produces air zones of different densities, which create different air movements. The kinetic energy of the wind can be used to drive turbine propellers that are capable of generating electricity. Some wind turbines are capable of producing up to $5 \mathrm{MW}$ of electricity, although they require a constant wind speed of about $5.5 \mathrm{~m} / \mathrm{s}$ or 20 kilometers per hour. In few areas of the Earth there are winds with constant speeds of this value, although stronger winds can be found at higher altitudes and in ocean areas.

The concept of "solar energy" refers to the energy that is directly produced by the transfer of sunlight radiated energy. It can be used to generate electricity or heat the air inside buildings. Although solar energy is renewable and easy to produce, the main problem is that the sun does not provide constant energy in any place on Earth. In addition, due to the rotation of the Earth around its axis and hence the day-night alternation, sunlight can't be used to generate electricity for a limited time each day. Another limitation of the use of this type of energy is the existence of cloudy days, when the solar energy capture potential decreases significantly due to the shielding of the Sun, limiting the applications of this form of renewable energy.
There is no disadvantage because solar installations bring benefits from all points of view.

The solar panels generate 9h/day of electricity (the calculation is minimal, the winter day is $9 \mathrm{~h}$ ). For $9 \mathrm{~h}$, these solar panels generate electricity and at the same time store energy in batteries for use at night.

Solar installations are of two types: thermal and photovoltaic. Photovoltaics produce electricity for free. Thermals help save gas $75 \%$ per year. A house that has both solar installations (with photovoltaic and vacuum thermal panels) is considered "NO FACTORS" because the energy accumulated in the day is spent in the network).

\section{Discussion}

Renewable energies are considered in practice, energies from sources that either regenerate by themselves in a short time, or are virtually inexhaustible sources. The term renewable energy refers to forms of energy produced by the energy transfer of energy from renewable natural processes. Thus, the energy of sunlight, winds, flowing waters, biological processes and geothermal heat can be captured by humans using different processes. Unrenewable energy sources include nuclear energy as well as the energy generated by the burning of fossil fuels, such as oil, coal and natural gas. These resources are obviously limited to the existence of these deposits and are generally considered nonrenewable. Renewable energy sources include:

- Wind energy usually expressed - wind energy

- Solar energy

- Water energy

- The hydraulic energy, the flowing water energy

- Tidal energy, sea/ocean flux/reflux energy

- Potential osmotic energy

- Geothermal energy, energy gained from the Earth's deep heat

- Biomass energy: Biodiesel, bioethanol, biogas

All these forms of energy are technically valuable and can be used to generate electricity, produce hot water, etc. Currently, they are unevenly exploited, but there is a clear and concrete trend that shows that there is an insistent investment in this relatively new energy branch.

Today we can hope to get infinite energy from the water through a simple, controlled process. It is about water split into hydrogen and oxygen by modern nano methods, using ultraviolet radiation, so that the energy used to separate water into its components being lower than that obtained then by burning hydrogen. Many experts work in this area and have already achieved remarkable results.

Once this process will have been put in place, it will allow water to be used in the future as an energy storage and storage agent. 


\section{High-Powered Stations}

Tidal energy is the energy that can be captured by exploiting the potential energy resulting from the vertical displacement of the water mass at different levels or the kinetic energy due to the tidal currents.

Tidal energy is a consequence of solar and lunar gravitational forces, as well as a consequence of ground rotation.

Large-scale power plants are of two types:

- Central without a dam

- Which uses only the kinetic energy of tidal current

- Central dam

- Which uses the potential energy created as a result of the water level rise during the flow

\section{Power Plants Using Wave Energy}

Waves are formed due to the action of moving air masses (wind) on the water. The magnitude of the waves generated depends on the wind speed, its duration and distance on the water (length of action).

The water movement resulting from this action has a kinetic energy exploitable with the help of special technologies.

Wave energy capture systems are of three types:

- The pressure piping system that is based on the fact that a pressure exerted on a large surface and transmitted by a liquid through pipes to a smaller surface multiplies the force on the surface unit and this force is used to drive an electric generator

- The system based on liquid ascension

- Which uses the rise of water as a wave on an artificial slope, then the turbine of an electric generator

- The liquid piston system

- Which is based on the fact that in an enclosure the waves produce by their climbing and lowering movement a Similar effect to a piston pumping or aspiring the air acting on the turbine of an electric generator

Potential osmotic energy or salinity gradient energy is the energy obtained from the salt concentration difference between sea water and river or river water. Two practical methods for this are reverse inverse Electrode (EDI) and delayed pressure Osmosis (OIP).

Both processes are based on osmosis with specific ion membranes, the resulting residual product being salty water.

The osmotic energy utilization is based on the osmotic pressure difference between freshwater and seawater (Fig. 13).

The first osmotic power plant, with a installed power of $4 \mathrm{~kW}$, was built in Norway by Statkraft and became operational on 24 November 2009. In this plant the energy is obtained on the basis of a flow rate of $10 \mathrm{l} / \mathrm{s}$ flowing through membrane at a pressure of 10 bar.

Increasing water flow and increasing pressure will increase the capacity to generate electricity.

\section{Disadvantages}

Drainage resulting as a residual product may have adverse effects on the environment.

Magnetic energy generators with no moving parts are based on permanent magnets coupled in different ways with ferromagnetic cores and whose magnetic field interacts with magnetic fields generated by electroniccontrolled coil groups (Fig. 14).

There are many such patented devices, systems and generators both scientists and amateurs over time. Among them, we mention:

- The electric generator built by Graham Gunderson

- Charles Flyn's device

- Lawrence Tseung's device

- Hans Coler's magneto generator

- The electromagnetic generator (MEG) built by Tom Bearden, Stephen Patrick, James Hayes, Kenneth Moore and James Kenny

- Thane Heins' transformer

- Richard Willis's generator

\section{Conclusion}

The analysis carried out in this paper highlights the fact that the evaluation of biomass sources is complex and the "resource potential" is a concept different from "providing available and sustainable resources". Territorial analysis often shows good availability of biomass, but if in the context of bioenergy production, only a small portion of available biomass can be used under sustainable conditions.

The biomass potential is not the same as available biomass and it is in turn different from sustainable biomass. Assessing the availability and sustainability of raw material is a critical issue in the strategic development of bio-energy projects and is closely linked to the selection of biomass technologies for energy generation.

Lignocellulosic biomass sources (both from energy crops and from residues or waste) are by far the most significant as spreading and may coexist rather synergistically than with other biomass uses such as food, materials, services Ecological and natural habitats.

The use of native biomass multi-species with longer rotation cycles represents the most significant opportunity to develop biomass resources that preserve biodiversity, carbon storage and ecosystems with minimal effect on food resources. Another aspect 
highlighted here is that each class of processing technologies is suitable for a certain range of chemical changes of the biomass constituents.

Thermal processing options are the most flexible of all technological classes and it is best to use the lignocellulosic biomass resources on a strategic scale. Processing technologies that produce both materials and energy that can be used on the market will be the ones that will meet the needs of society and open up sustainable business opportunities.

A carbon neutral future will still require significant carbon-based resources such as liquid transport fuels and organic chemicals so that thermal processing technologies from which multiple products are obtained are preferred. A sustainable bioenergy pathway involves extracting the maximum value from the biomass resource.

Typically, biomass products that use the resource's primary material qualities are of greater value. This is why pre-processing for the extraction of wood, oil, protein and solid sugars (if economically convenient) is encouraged and this should be seen as a support for bioenergy production.

The development of specific bioenergy production projects is not therefore based solely on economic conditions, but must also take into account social and environmental aspects. If the factors considered are properly addressed, biomass or bioenergy production projects must first be judged through a specific technical and economic modeling, preceded by pre-feasibility studies, life cycle assessment and feasibility studies.

Today we can hope to get infinite energy from the water through a simple, controlled process. It is about water split into hydrogen and oxygen by modern nano methods, using ultraviolet radiation, so that the energy used to separate water into its components being lower than that obtained then by burning hydrogen. Many experts work in this area and have already achieved remarkable results.

Once this process will have been put in place, it will allow water to be used in the future as an energy storage and storage agent.

\section{Acknowledgment}

The work was appreciated by teams of professors from the departments of automobiles from several universities in Romania and Italy. This text was acknowledged and appreciated by Associate Professor Aniello Riccio SECONDA UNIVERSITA' DEGLI STUDI DI NAPOLI Italy, Dr. (Ms.) Shweta Agarwala Senior Research Scientist at Singapore Center for 3D Printing Nanyang Technological University Singapore, whom we thanks and in this way.

\section{Author's Contributions}

All the authors contributed equally to prepare, develop and carry out this manuscript.

\section{Ethics}

This article is original. Authors declare that are not ethical issues that may arise after the publication of this manuscript.

\section{References}

Aversa, R., R.V.V. Petrescu, A. Apicella and F.I.T. Petrescu, 2017a. Nano-diamond hybrid materials for structural biomedical application. Am. J. Biochemistry Biotechnol.

Aversa, R., R.V. Petrescu, B. Akash, R.B. Bucinell and J.M. Corchado et al., 2017b. Kinematics and forces to a new model forging manipulator. Am. J. Applied Sci., 14: 60-80.

Aversa, R., R.V. Petrescu, A. Apicella, F.I.T. Petrescu and J.K. Calautit et al., 2017c. Something about the $\mathrm{V}$ engines design. Am. J. Applied Sci., 14: 34-52.

Aversa, R., D. Parcesepe, R.V.V. Petrescu, F. Berto and G. Chen et al., 2017d. Processability of bulk metallic glasses. Am. J. Applied Sci., 14: 294-301.

Aversa, R., F.I.T. Petrescu, R.V. Petrescu and A. Apicella, 2016a. Biomimetic FEA bone modeling for customized hybrid biological prostheses development. Am. J. Applied Sci., 13: 1060-1067. DOI: 10.3844/ajassp.2016.1060.1067

Aversa, R., D. Parcesepe, R.V. Petrescu, G. Chen and F.I.T. Petrescu et al., 2016b. Glassy amorphous metal injection molded induced morphological defects. Am. J. Applied Sci., 13: 1476-1482.

Aversa, R., R.V. Petrescu, F.I.T. Petrescu and A. Apicella, 2016c. Smart-factory: Optimization and process control of composite centrifuged pipes. Am. J. Applied Sci., 13: 1330-1341.

Aversa, R., F. Tamburrino, R.V. Petrescu, F.I.T. Petrescu and M. Artur et al., 2016d. Biomechanically inspired shape memory effect machines driven by muscle like acting NiTi alloys. Am. J. Applied Sci., 13: 1264-1271.

'Blue Planet' - the general culture magazine.

DiPippo, R., 2004. Second law assessment of binary plants generating power from low-temperaturegeothermal fluids. Geothermics, 33: 565-586.

Giovanni, R., E. Foppapedretti and C.D. Carolis, 2012. Manual - renewable sources.

Halliday, D. and R. Robert, 1966. Physics, Part II. 1st Edn., John Wiley and Sons, Inc., New York.

Hydrogen, from Wikipedia. The free encyclopedia. 
Krane, K.S., 1987. Introductory Nuclear Physics. 3rd Edn., Wiley and Sons, New York, ISBN-10: 047180553X, pp: 864 .

Lindal, B., 1973. Industrial and other Application of Geothermal Energy. In: Geothermal Energy, Armstead, H.C.H., (Ed.), UNESCO, Paris, pp: 135-148.

Mirsayar, M.M., V.A. Joneidi, R.V.V. Petrescu, F.I.T. Petrescu and F. Berto, 2017. Extended MTSN criterion for fracture analysis of soda lime glass. Eng. Fracture Mechan., 178: 50-59. DOI: $10.1016 /$ j.engfracmech.2017.04.018

Petrescu, F.I., 2012. Cold nuclear fusion. Plasma Physics and Fusion Technology (S70), INIS.

Petrescu, F.I. and J.K. Calautit, 2016a. About nano fusion and dynamic fusion. Am. J. Applied Sci., 13: 261-266. DOI: 10.3844/ajassp.2016.261.266

Petrescu, F.I. and J.K. Calautit, 2016b. About the light dimensions. Am. J. Applied Sci., 13: 321-325. DOI: 10.3844/ajassp.2016.321.325

Petrescu, F.I. and R.V. Petrescu, 2012. News in Physics. 1st Edn., Books on Demand, ISBN-13: 978-3848229642, pp: 82.

Petrescu, R.V.V., R. Aversa, A. Apicella, F. Berto and $\mathrm{S}$. Li et al., 2016a. Ecosphere protection through green energy. Am. J. Applied Sci., 13: 1027-1032. DOI: 10.3844/ajassp.2016.1027.1032
Petrescu, F.I.T., A. Apicella, R.V.V. Petrescu, S.P. Kozaitis and R.B. Bucinell et al., 2016b. Environmental protection through nuclear energy. Am. J. Applied Sci., 13: 941-946.

Progress in Fusion, ITER.

Sanner, B., C. Karytsas, D. Mendrinos and L. Rybach, 2003. Current status of ground source heat pumps and underground thermal energy storage in Europe. Geothermics, 32: 579-588. DOI: 10.1016/S03756505(03)00060-9

Stacey, F.D. and D.E. Loper, 1988. Thermal history of the Earth: A corollary concerning non-linear mantle rheology. Physics Earth Planetary Interiors, 53: 167-174.

Shultis, J.K. and R.E. Faw, 2002. Fundamentals of Nuclear Science and Engineering. CRC Press, New York, ISBN-10: 0824708342, pp: 520.

Thermonuclear Weapon, from Wikipedia. The free encyclopedia.

World Tree, From Wikipedia. Free Encyclopedia.

\section{Source of Figures:}

Fig. 11: http://www.energiialternative.net/hidro2.htm

Fig. 12: http://www.energiialternative.net/hidro3.htm

Fig. 13: http://www.energiialternative.net/altaer.htm

Fig. 14: http://www.energiialternative.net/magmls.htm 